\title{
Essential role of the RNA-binding protein HuR in progenitor cell survival in mice
}

\author{
Mallika Ghosh, ${ }^{1}$ Hector Leonardo Aguila, ${ }^{2}$ Jason Michaud, ${ }^{1}$ Youxi Ai, ${ }^{1}$ Ming-Tao Wu, ${ }^{1}$ \\ Annabrita Hemmes, ${ }^{3}$ Ari Ristimaki, 3,4 Caiying Guo, ${ }^{5}$ Henry Furneaux, ${ }^{1,6}$ and Timothy Hla ${ }^{1,7}$

\begin{abstract}
${ }^{1}$ Center for Vascular Biology and 2Department of Immunology, University of Connecticut Health Center, Farmington, Connecticut, USA. ${ }^{3}$ Department of Pathology, HUSLAB, and Haartman Institute, Helsinki University Central Hospital, and Genome-Scale Biology Research Program, University of Helsinki, Helsinki, Finland. "Department of Pathology, Division of Diagnostics, University of Oulu, and Oulu University Hospital, Oulu, Finland. 5Janelia Farm Campus, Howard Hughes Medical Institute, Reston, Virginia, USA.

${ }^{6}$ Department of Molecular, Microbial, and Structural Biology, University of Connecticut Health Center, Farmington, Connecticut, USA. ${ }^{7}$ Center for Vascular Biology, Department of Pathology and Laboratory Medicine, Weill Cornell Medical College, New York, New York, USA.
\end{abstract}

\begin{abstract}
The RNA-binding protein HuR (also known as ELAV1) binds to the $3^{\prime}$-untranslated region of mRNAs and regulates transcript stability and translation. However, the in vivo functions of $\mathrm{HuR}$ are not well understood. Here, we report that murine $\mathrm{HuR}$ is essential for life; postnatal global deletion of Elavl1 induced atrophy of hematopoietic organs, extensive loss of intestinal villi, obstructive enterocolitis, and lethality within 10 days. Upon Elavl1 deletion, progenitor cells in the BM, thymus, and intestine underwent apoptosis, whereas quiescent stem cells and differentiated cells were unaffected. The survival defect of hematopoietic progenitor cells was cell intrinsic, as transplant of Elavl1 ${ }^{-/}$BM led to compromised hematopoietic reconstitution but did not cause lethality. Expression of p53 and its downstream effectors critical for cell death were induced in progenitor cells as HuR levels declined. In mouse embryonic fibroblasts, HuR bound to and stabilized the mRNA for $M d m 2$, a critical negative regulator of $\mathrm{p} 53$. Furthermore, cell survival was restored by expression of $\mathrm{Mdm} 2$ in Elavl1 ${ }^{-/-}$cells, suggesting that HuR keeps p53 levels in check in progenitor cells and thereby promotes cell survival. This regulation of cell stress response by HuR in progenitor cells, which we believe to be novel, could potentially be exploited in cytotoxic anticancer therapies as well as stem cell transplant therapy.
\end{abstract}

\section{Introduction}

In eukaryotes, once mRNA precursors are transcribed, splicing, transport, translation, and degradation are carefully regulated to achieve precise spatial, temporal, and tissue-specific control of gene expression $(1,2)$. The significance of such mechanisms, collectively known as posttranscriptional RNA regulation, in physiology and pathology is just beginning to be appreciated. Current data support a model whereby specific RNA-binding proteins and small RNAs (such as microRNA [miRNA]) interact with noncoding, regulatory regions of mRNAs and determine the fate of a given transcript $(3,4)$.

HuR (also known as ELAV1), a member of the ELAV (embryonic lethal abnormal vision) RNA-binding protein family, plays an important role in AU-rich element-mediated (ARE-mediated) mRNA stability and translation (5-7). HuR function is closely tied to its translocation from the nucleus into the cytoplasm, in which it modulates transcript stability and translation.

In vitro studies have shown that $\mathrm{HuR}$ regulates expression of genes involved in key cellular processes, such as tumorigenesis, hypoxic gene regulation, cell-cycle control, inflammation, cell stress response, and apoptosis (5-9). Indeed, HuR promotes survival of cells and positively regulates the expression of antiapoptotic proteins and suppresses proapoptotic proteins (10-12). Enhanced HuR expression is correlated with cancer progression (13-15), thus serving as a prognostic biomarker for adenocarcinoma.

In contrast, in vivo function of HuR is not clear, as only limited number of studies have examined the function of this RNAbinding protein in animal models. When HuR was overexpressed in the myeloid lineage in a transgenic mouse model, it induced

Conflict of interest: The authors have declared that no conflict of interest exists. Citation for this article: J. Clin. Invest. 119:3530-3543 (2009). doi:10.1172/JCI38263. translational silencing of the ARE-containing transcripts, such as TNF- $\alpha$, even though the mRNAs were stabilized (16). Global overexpression of HuR in transgenic mice led to impairment of male gametogenesis, suggesting a role of HuR in germ cell function (17). A recent study published during the review of this manuscript reported the essential role of HuR in mouse embryogenesis. Elavl1 null mice exhibited embryonic lethality due to defects in placental development. In addition, defects in spleen and bone development were also observed, suggesting the complex role of HuR in embryonic development (18).

With the aim of understanding the in vivo function of $\mathrm{HuR}$, we developed a mouse model in which the Elavl1 gene can be deleted by tamoxifen-inducible Cre recombinase-mediated gene excision. We report for what we believe to be the first time the essential role of posttranscriptional regulator HuR in the survival of progenitor cell populations in hematopoietic and intestinal systems. We also describe a HuR-regulated mechanism in progenitor cells that keeps p53 protein levels in check. These data underscore the essential role of posttranscriptional gene regulatory mechanisms in progenitor cell fate in vivo.

\section{Results}

Essential postnatal role of $H u R$. The schematic of the Elavl1f/f construct in which the Elavl1 gene can be deleted by Cre-Lox-mediated gene excision is shown in Figure 1A. We crossed Elavl1 ${ }^{f /+}$ mice with Hprt-Cre mice (19), which yielded Elavl1 ${ }^{+/-}$mice. Intercrosses of F1 generation and analysis of approximately $300 \mathrm{~F} 2$ mice yielded Elavl1 $1^{+/-}$and Elavl1 $1^{++}$mice in the ratio of 2:1. However, no liveborn Elavl1 $1^{-/-}$mice were derived, indicating that Elavl1 is required for normal embryonic development. Elavl1 ${ }^{+/}$mice appeared healthy and were able to reproduce. 
A

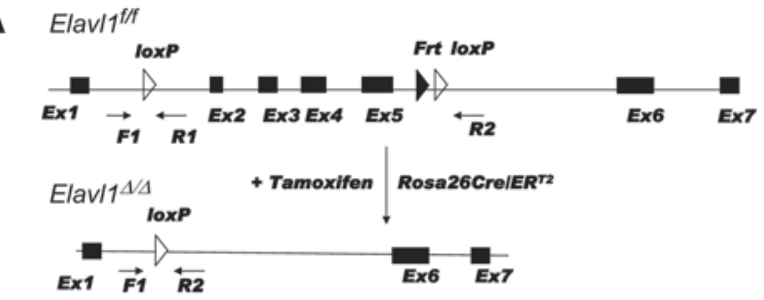

C

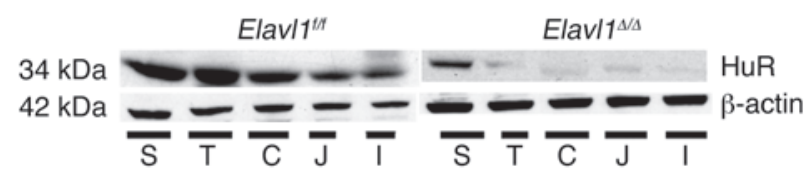

E
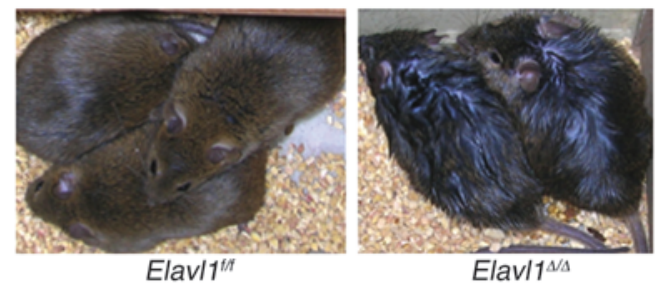

B

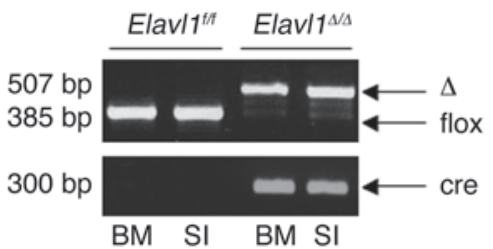

D
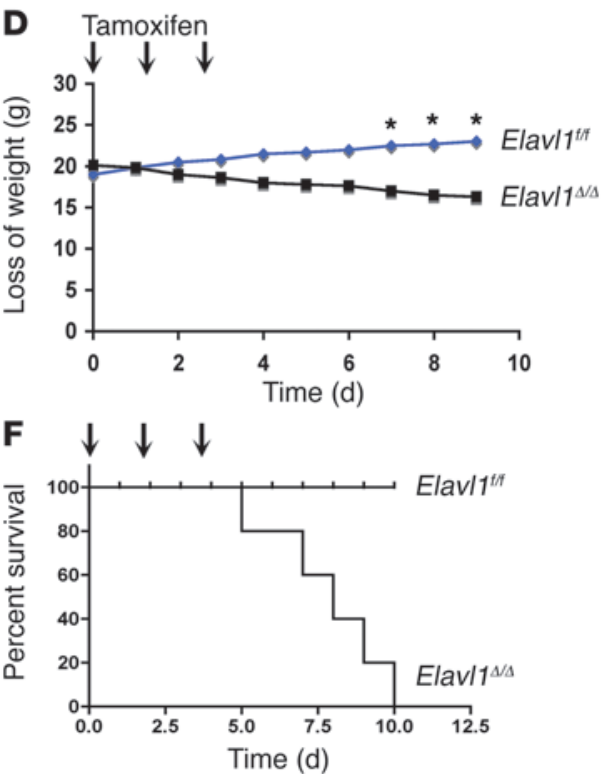

\section{Figure 1}

Deletion of Elav/1 in adult mice leads to lethality. (A) Tamoxifen-induced Elav/1 disruption in Elavl1/f/Rosa26Cre/ERT2 (Elav/1 ${ }^{\Delta / \Delta}$ ) mice. A schematic representation of conditional Elavl1 gene locus is shown. Arrows indicate the position of PCR primers; open triangles indicate loxP sites; the filled triangle indicates Frt site; and filled boxes indicate exons. F1, forward 1; R1, reverse 1. (B) PCR analysis of genomic DNA from indicated organs of mice 4 days after tamoxifen administration indicates either floxed or Elav/1 deletion $(\Delta)$ or cre bands. SI, small intestine. (C) IB analysis of tissue extracts with HuR antibody from Elav/1 $1^{t / f}$ and Elav/1 ${ }^{\Delta / \Delta}$ mice, 3 days after tamoxifen treatment. The same blot was reprobed with a $\beta$-actin mouse mAb. S, spleen; T, thymus; C, colon; J, jejunum; I, ileum. (D) Weight loss monitored over a period of 10 days upon deletion of Elavl1. $\left(n=4 ;{ }^{\star} P<0.05\right)$. (E) Cachexic phenotype of Elav/1 ${ }^{\Delta / \Delta}$ mice observed 4 days after tamoxifen administration. (F) Kaplan-Meier survival curves of Elav/1 $1^{f / f}[n=8]$ and Elav/1 ${ }^{\Delta / \Delta}[n=10]$ mice after tamoxifen treatment $(P<0.01$, Log-rank test). Arrows indicate 3 doses of tamoxifen administration in $\mathbf{D}$ and $\mathbf{F}$.

We then proceeded to determine the postnatal function of HuR by tamoxifen-inducible Cre (Rosa26Cre/ER ${ }^{\mathrm{T} 2}$ ) recombinase-mediated gene deletion (20). Elavl1f/fRosa26Cre/ERT2 mice (8 weeks old) were administered tamoxifen (by oral administration for 3 consecutive days), and the extent of deletion of the Elavl1 gene was determined by PCR of genomic DNA (the resulting mouse strain is referred to as Elavl $1^{\Delta / \Delta}$ ). Analysis at 4 days showed that the Elavl1 gene was deleted efficiently by tamoxifen administration (Figure 1B), with concomitant reduction of HuR polypeptide expression in intestinal and hematopoietic tissues (Figure 1C). After tamoxifen administration, Elavl $1^{\Delta / \Delta}$ mice exhibited signs of cachexia and eventually died within 10 days, whereas the Elavl1 f/f mice were unaffected (Figure 1, D-F). These data indicate that HuR function is essential for postnatal life.

Lack of HuR leads to hematopoietic and immune defects. Rapid and dramatic atrophy of immune and hematopoietic organs were observed upon Elavl1 deletion (Figure 2A). Histological sections indicate marked decrease in cellularity, with disappearance of follicles in these organs. In the BM sections, decreased cellularity of immature cells was observed (Figure 2B). Total cell numbers in BM, thymus (Figure 2C), spleen, and LN (data not shown) declined rapidly, sug- gesting a profound effect on hematopoiesis. In blood, leukocyte counts declined by approximately $65 \%$ within 4 days. In contrast, granulocyte counts were slightly increased, approximately 2 fold, while platelets and red blood cell numbers remained the same (Supplemental Figure 1; supplemental material available online with this article; doi:10.1172/JCI38263DS1), suggesting that HuR function may be more critical in actively proliferating cells.

To define the cellular changes in the hematopoietic lineages, we performed a detailed flow cytometric analysis of cells from Elavl1f/f and Elavl1 ${ }^{\Delta / \Delta}$ mice. For the BM, we evaluated the presence of HSCs $(21,22)$, common lymphoid progenitors (CLPs) (23), total B cells, immature B cells, mature B cells (24), myeloid cells and their progenitors $(25,26)$, and erythroid progenitors (27) (Figure 3, A and B). These results showed an overall maintenance of HSCs, decreased numbers of CLPs, B cell progenitors, myeloid cells and their progenitors, and erythroid progenitors. Mature populations were not affected, indicating that progenitor cells are selectively targeted. This was validated by ex vivo hematopoiesis assays in methylcellulose (Figure 3C), showing a decreased number of myeloerythroid colonies in BM from the HuR-depleted animals. 
A

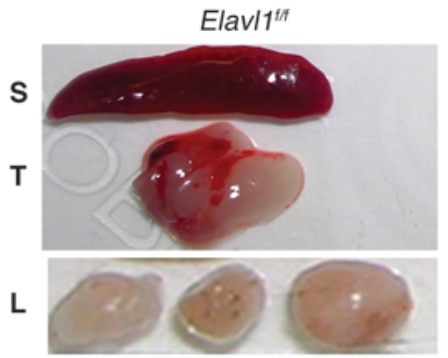

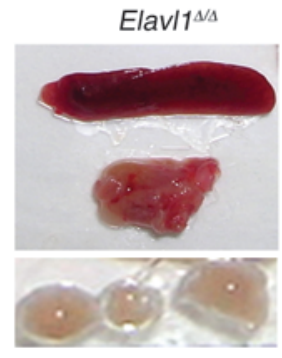

B

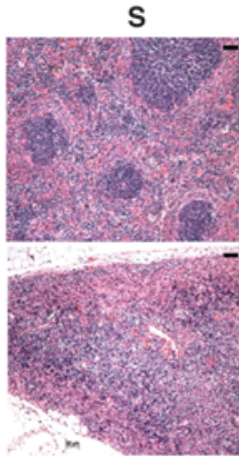

T

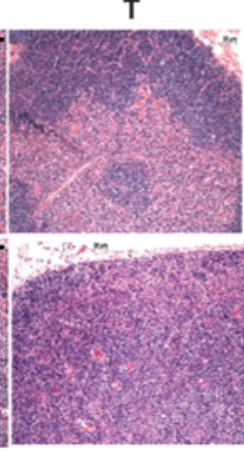

L

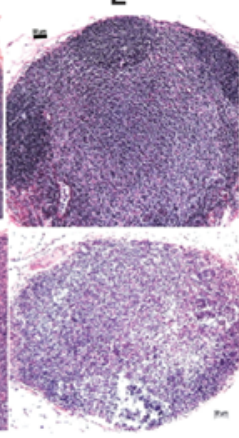

B

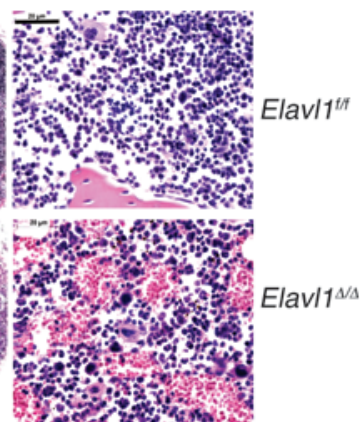

C

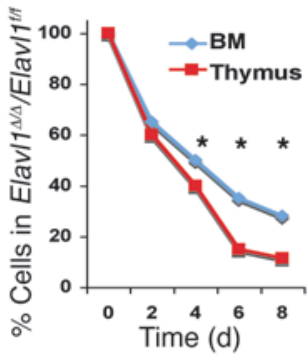

Figure 2

Requirement of HuR for postnatal hematopoiesis. (A) Atrophy of spleen, thymus, and LN (L) 4 days after deletion of Elav/1. (B) H\&E stain of tissue sections from Elav/1//f or Elav/1 $1 / \Delta$ mice 4 days after tamoxifen treatment. Original magnification, $\times 5$ (spleen, thymus, and LN); $\times 40$ (BM). Scale bar: $50 \mu \mathrm{m}$ (spleen, thymus, and LN); $20 \mu \mathrm{m}$ (bone). B, BM. (C) Elav/1/f/f and Elav/1 ${ }^{\Delta / \Delta}$ mice were treated with tamoxifen, and the total number of BM cells and thymocytes were counted at indicated times $\left({ }^{*} P<0.05 ; n=5\right)$.

To determine the effect of HuR on T cell lineage, we evaluated the distribution of cells in different stages of thymocyte development (Figure 3B) (28). Results show selective depletion of doublepositive $\mathrm{T}$ cells, which are known to be rapidly proliferating (Figure 3B). However, single-positive (CD4 ${ }^{+}$and $\left.\mathrm{CD}^{+}\right)$and double-negative (CD4- and $\left.\mathrm{CD}^{-}\right)$populations showed an increase in percentage cellularity due to decreased cell numbers (Figure 2C).

Regulation of hematopoietic progenitor cell survival and proliferation by $H u R$. To understand the underlying cause of decreased cellularity, we measured apoptosis and necrosis by flow cytometry (29). Analysis of BM cells showed increased apoptotic and necrotic cells in the $B$ progenitor population (Figure $4, A$ and $B$ ). In contrast, mature $B$ cells did not exhibit increased cell death upon the deletion of Elavl1. Interestingly, BrdU incorporation was also significantly attenuated in the B cell population (Figure $4 C$ ). Immunohistochemistry with BrdU antibody confirmed these results (Supplemental Figure 2). In thymus, increased apoptosis and necrosis and decreased BrdU incorporation in the double-positive $\mathrm{T}$ progenitor $\left(\mathrm{CD}^{+} \mathrm{CD}^{+}\right)$ population (Figure 4, D-F) was observed upon Elavl1 deletion. These data strongly suggest that $\mathrm{HuR}$ is required for the survival of progenitor cells in the BM and the thymus.

Next, HuR expression during early hematopoiesis and B cell development was determined by immunofluorescence analysis of FACS-sorted BM-derived cells. Low HuR staining was detected in the cytoplasm and nucleus of quiescent long-term HSC (LT-HSC) population $\left(\mathrm{Lin}^{-} \mathrm{Sca}-1^{+} \mathrm{c}-\mathrm{kit}^{+} \mathrm{Flt3} 3^{-}\right)$(Figure 4G) (30). In the shortterm HSC (ST-HSC) population $\left(\mathrm{Lin}^{-} \mathrm{Sca}-1^{+} \mathrm{C}-\mathrm{kit}^{+} \mathrm{Flt} 3^{+}\right)$, which included early progenitors, strong HuR staining was seen in more than $90 \%$ of cells. Interestingly, HuR staining in this population highlights punctate structures in the nucleus. In the CLP population ( $\mathrm{Lin}-\mathrm{Sca}-1^{+} \mathrm{c}-\mathrm{kit} \mathrm{IL}^{+} \mathrm{IL}-7 \mathrm{R}^{+}$), a similar pattern of HuR expression was seen; however, cells expressing lower levels of HuR were also observed. In the immature B cell population ( $\left.\mathrm{CD}^{2} \mathrm{R}^{+} \mathrm{IgM}^{-}\right)$, only $30 \%$ of the cells expressed HuR in the cytoplasm. In the mature B cell population, primarily cytoplasmic localization was seen in approximately $20 \%$ of the cells.

The specificity of Cre-mediated Elavl1 gene deletion in different cell populations was tested by analyzing FACS-sorted cells. As shown in Figure 4H, Elavl1 mRNA expression is markedly reduced in both HSC and early lymphoid progenitor (ELP) populations. $\mathrm{BrdU}^{+}$cells are prominent in the ELP population and drastically reduced by Elavl1 gene deletion (Figure 4I). Cell-cycle analysis of the sorted progenitor population indicated that Elavl1 gene deletion did not influence the cell-cycle parameters (Supplemental Figure 3), suggesting that the primary effect of HuR loss is the induction of apoptosis.

$H u R$ maintains intestinal integrity by preventing epithelial progenitor cell apoptosis. Because the hematopoietic and immune deficiency alone is unlikely to be the main cause of lethality in the time frame of the experiment ( $<10$ days), we examined other organ systems after Elavl1 deletion. The gastrointestinal (GI) tract exhibited marked abnormality within 2-4 days of Elavl1 deletion. As shown in Figure 5A, the stomach was distended and the proximal intestine was devoid of food, suggesting intestinal blockage. In contrast to the GI tract, gross morphology and histological sections of skin, heart, liver, kidney, lung, and mammary gland appeared normal, even though effective reduction in HuR polypeptide was seen in these tissues (Supplemental Figure 4). Moreover, induction of vascular collapse and hypoxia in the affected tissues were ruled out, because hypoxia-inducible factor- $1 \alpha$ expression was not induced after HuR deletion (Supplemental Figure 5). Histological analysis of the small intestine (Figure 5B) indicated exten- 


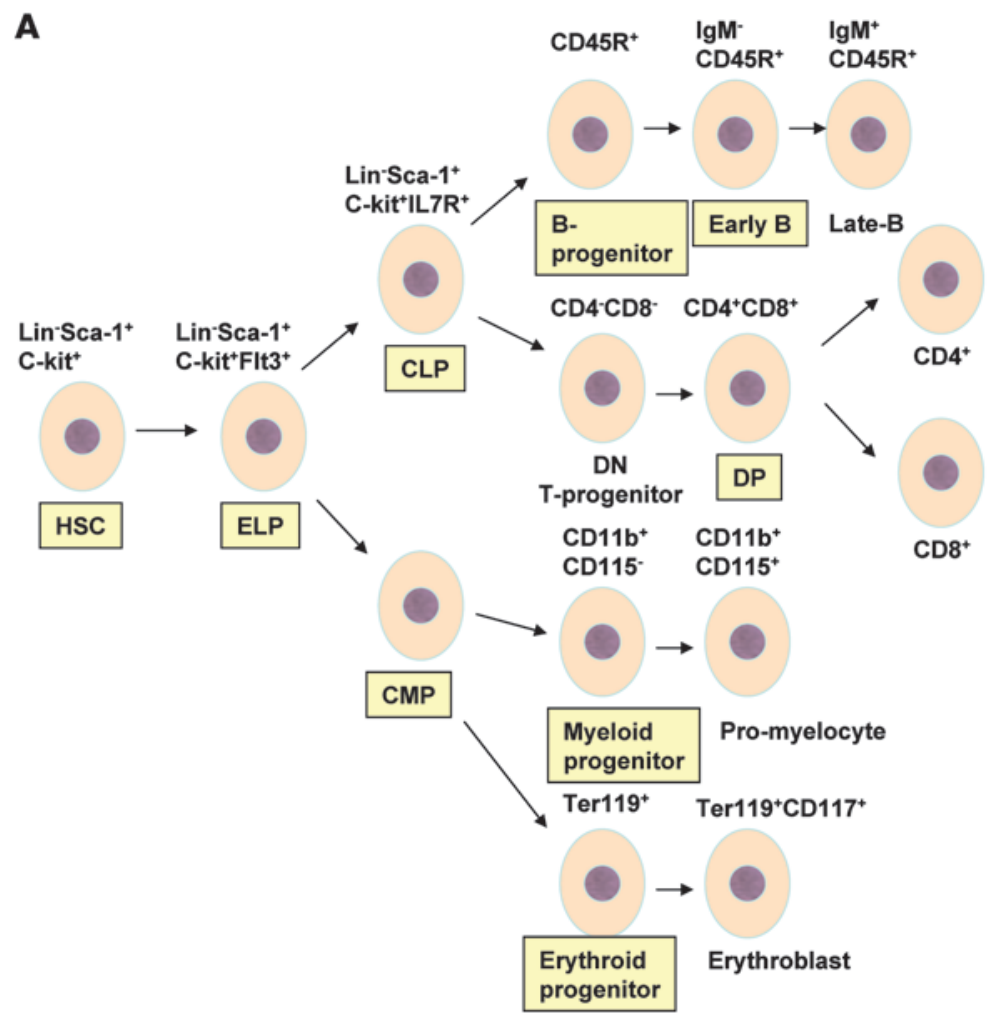

B
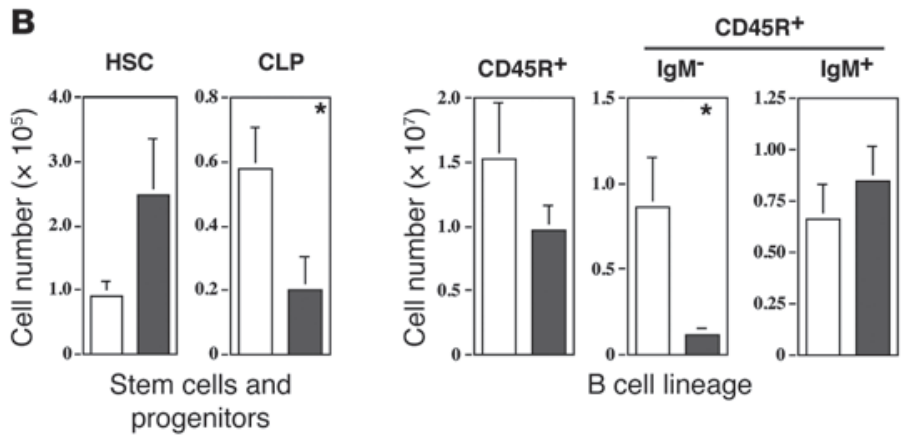

CD11b+
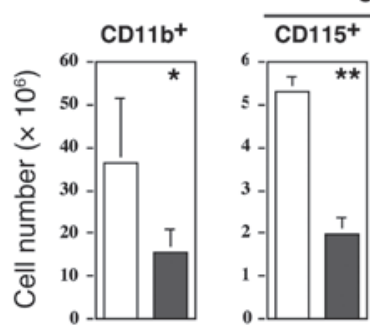

Myeloid lineage

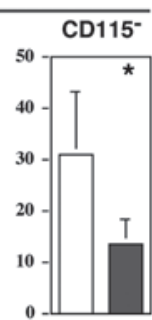

0

$\mathrm{CD}^{+}$

$\mathrm{T}$ cell lineage

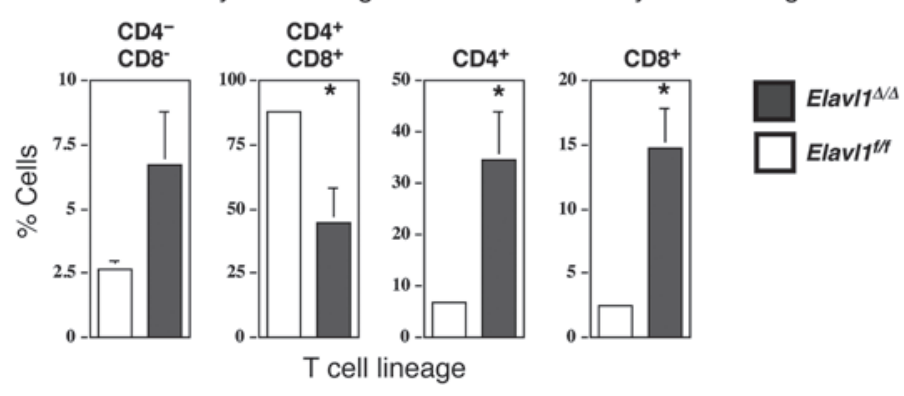

C

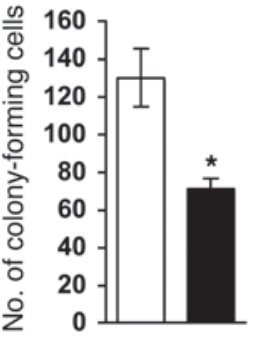

\section{Figure 3}

Effect of HuR loss on hematopoietic cells. (A) A schematic of hematopoietic cell lineage and their markers (CMP, common myeloid progenitor; DN, double negative; DP, double positive). Cells that were affected by loss of Elavl1 are shaded. (B) Distribution of HSCs (Lin-CD117+Sca-1+), CLPs (Lin-CD117+IL-7R $\alpha^{+}$), total B cells (B220/CD45R ${ }^{+}$CD19 ${ }^{+}$), immature $B$ cells $\left(C D 19+B 220^{+} \operatorname{lgM}-C D 117^{+}\right)$, mature $B$ cells $\left(B_{22} 0^{+} \mathrm{gM}^{+}\right)$, myeloid cells (CD11 $\left.\mathrm{b}^{+}\right)$, monocytes/ macrophages (CD11 $b^{+}$CD115+), and erythroid progenitors (Ter119+, $\mathrm{CD}_{117^{+}}$) in BM. Cells were quantified by multiparameter flow cytometry and were analyzed 4 days after tamoxifen administration of Elav/1 ${ }^{1 / f}$ and Elavl $1^{\Delta / \Delta}$ mice $\left({ }^{\star} P<0.05\right.$, $\left.{ }^{\star \star} P<0.01 ; n=4\right)$. (C) Methylcellulose CFC assay of total BM cells isolated from Elav/1 $1^{\mathrm{flf}}$ and Elav/1 ${ }^{\mathrm{s} / \mathrm{A}}$ mice, 4 days after tamoxifen treatment, and grown for 11 days in vitro ( $\left.{ }^{\star} P<0.05 ; n=4\right)$. 


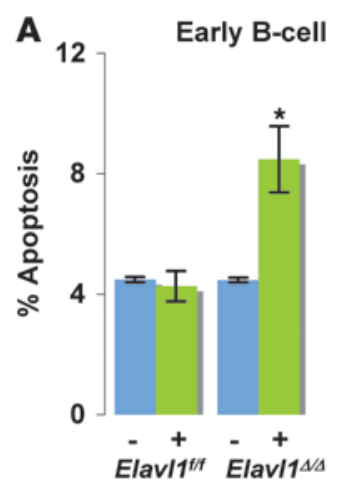

C
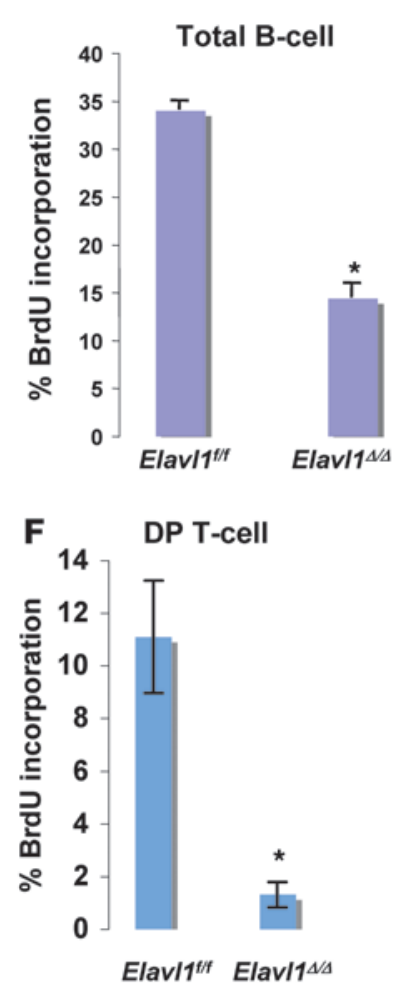

H

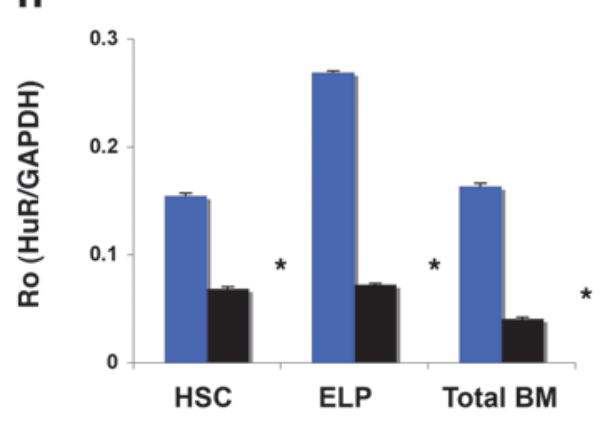

G
B Early B-cell

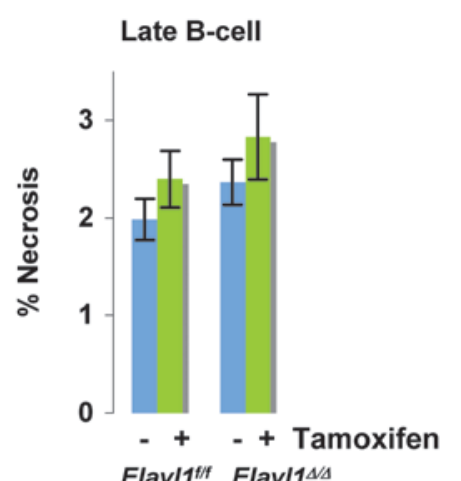

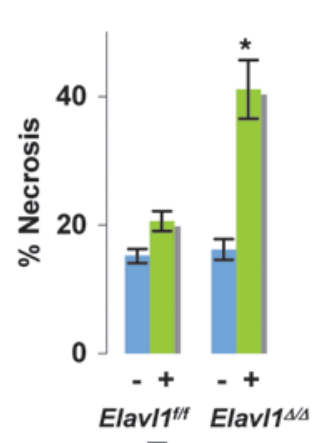

E
DP T-cell
D

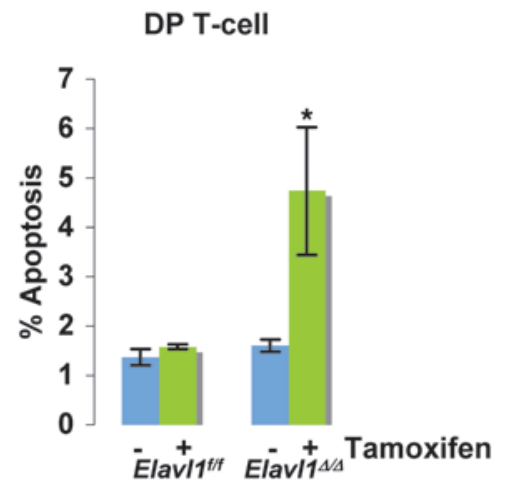

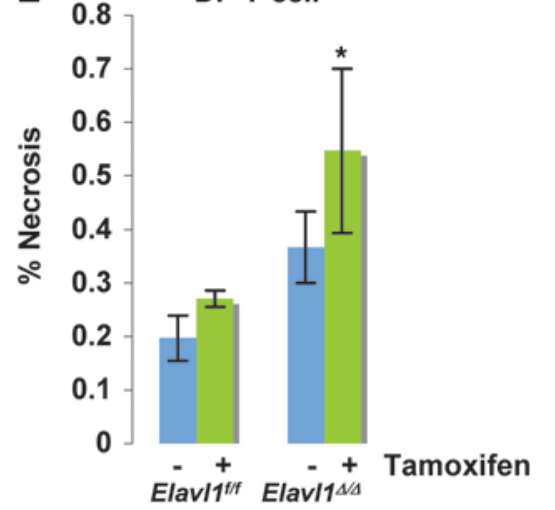
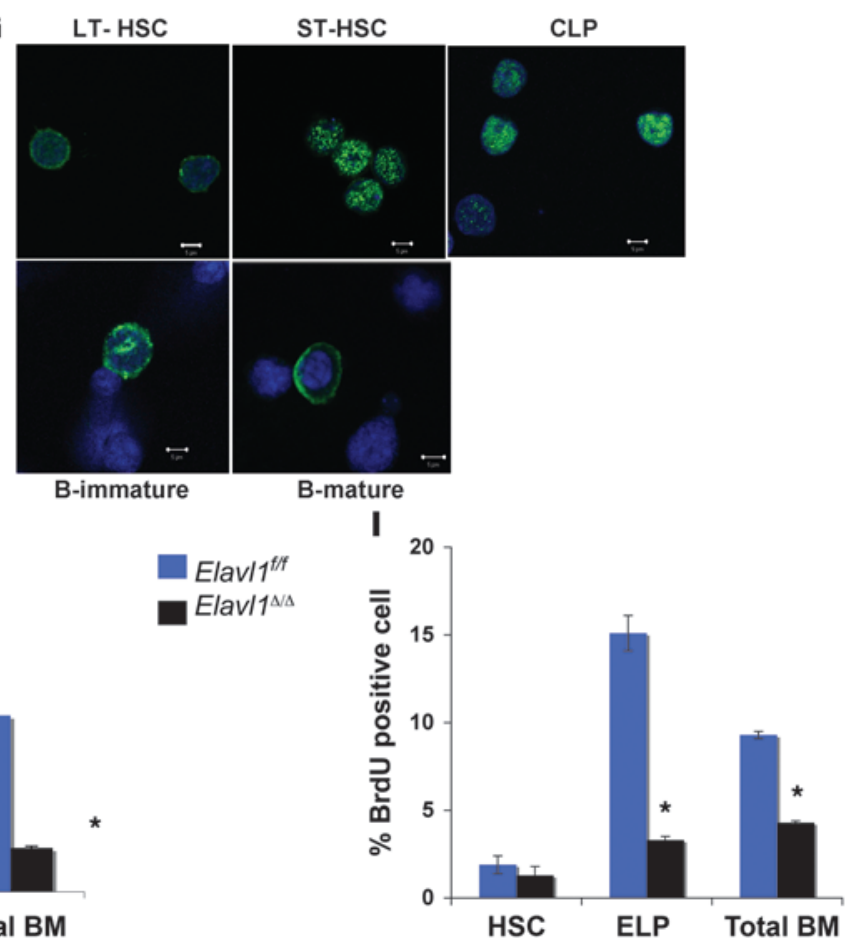


\section{Figure 4}

Regulation of immune and hematopoietic progenitor cell survival and proliferation by HuR. (A and B) BM cells from Elav/1 ${ }^{f / f}$ and Elav/1 $1 / \Delta$ mice were analyzed by flow cytometry to detect $B$ cell progenitors $\left(\mathrm{CD} 19^{+} \mathrm{CD} 45 \mathrm{R} / \mathrm{B} 220^{\text {hil }} \mathrm{gM}^{-} \mathrm{CD} 117^{+}\right)$and mature $\mathrm{B}$ cells $\left(\mathrm{CD} 19+\mathrm{CD} 45 \mathrm{R} / \mathrm{B} 220^{\mathrm{hi}} \operatorname{lgM}{ }^{+} \mathrm{CD} 117^{+}\right)$that are undergoing apoptosis (A) and necrosis (B) 48 hours after tamoxifen treatment $\left({ }^{\star} P<0.05 ; n=3\right)$. (C) BrdU incorporation into B cells $\left(\mathrm{B}^{2} 20^{+} \operatorname{lgM}{ }^{-}\right)$ was detected by flow cytometry 48 hours after tamoxifen treatment $\left({ }^{*} P<0.05 ; n=3\right)$. (D) Thymocytes from Elav/1/ff and Elav/1 ${ }^{\mathrm{A} / \Delta}$ mice were analyzed by flow cytometry to detect double-positive $T$ progenitors $\left(\mathrm{CD} 4^{+} \mathrm{CD} 8^{+}\right)$that are undergoing apoptosis $(\mathrm{E})$, necrosis $(F)$, and proliferation 48 hours after tamoxifen treatment $\left({ }^{*} P<0.05\right.$; $n=3)$. (G) HuR expression in BM-derived hematopoietic cell populations sorted by FACS was detected by immunofluorescence confocal microscopy. HuR (green); nucleus (blue). Original magnification, $\times 63$. Scale bar: $5 \mu \mathrm{m}$. (H) Elavl1 mRNA expression and (I) percentage of BrdU-positive cells in BM-derived hematopoietic cell populations sorted by FACS isolated from Elav/1 $1^{f / f}$ and Elav/1 ${ }^{\Delta / \Delta}$ mice 48 hours after tamoxifen treatment by q-RT-PCR. ( $\left.{ }^{\star} P<0.05 ; n=3\right)$. Ro indicates mRNA levels.

sive villus atrophy and disruption of the epithelial architecture within 2-3 days following gene deletion. In the colon, epithelial disruption at the mucosal layer and goblet cell loss were observed. These observations suggest that HuR function is essential for intestinal turnover and/or homeostasis.

In the small intestine, HuR is expressed in both epithelial and stromal compartments (Figure 5C). Nuclear HuR was detected strongly in the proliferating progenitors in the crypts, mature epithelial cells, and few stromal cells in the lamina propria. HuR expression was weaker in the terminally differentiated epithelial cells in the tip of the villus. When Elavl1 gene was deleted by tamoxifen administration, proliferation of progenitor cells in the crypts decreased (Figure 5C). In addition, TUNEL staining indicated significant increase in apoptotic cells (WT, $0.5 \%$; $\mathrm{KO}$, $14.3 \%, n=3 ; P=0.015$; Figure 5C). Likewise, in the colon (Figure $5 \mathrm{D})$, apoptosis of cells in the lamina propria was increased upon the deletion of Elavl1 (WT, $12.12 \pm 3.32, n=2$; KO, $42.88 \pm 8.45$, $n=8 ; P=0.0019)$. Epithelial cell apoptosis was also observed (WT, $1.38 \pm 0.53, n=3 ; \mathrm{KO}, 4.88 \pm 0.79, n=8 ; P=0.0030)$. Furthermore, lack of HuR suppressed $\beta$-catenin and E-cadherin expression in the intestinal epithelium, suggesting that HuR expression may be required to maintain the expression of key regulatory proteins required for the integrity of the epithelial layer (Figure 5E). Staining of the pan-macrophage marker F4/80 was not significantly altered after Elavl1 deletion (Figure 5F). These data suggest that $\mathrm{HuR}$ is required for proliferation and integrity of the intestinal epithelial progenitor cells.

Increased sensitivity to irradiation in Elavl1 ${ }^{+/-}$mice. Since the phenotype of the Elavl1 $\mathrm{KO}$ mice appears to resemble various aspects of lethal radiation syndrome, such as BM suppression and intestinal injury, we asked whether loss of an allele of Elavl1 leads to increased sensitivity to radiation injury. Nonirradiated Elavl1 ${ }^{+/-}$ mice had slightly reduced number of hematopoietic cells in BM and spleen compared with the Elavl1 ${ }^{+/+}$mice (Figure 6, B and C). When the mice were subjected to whole-body gamma irradiation ( 9 Gy, which is sufficient to induce BM suppression but not intestinal injury), the decrease in cellularity in the BM, spleen, and thymus of Elavl1 ${ }^{+/-}$mice was much more drastic (Figure 6, A, C, and D). Tamoxifen treatment of Elav1 ${ }^{w t / w t} R o s a C r e^{E R T 2}$ mice did not result in loss of cellularity in BM and thymus, which ruled out Cremediated toxicity (Supplemental Figure 6). Thus, HuR protects progenitor cells from gamma irradiation-induced cell death.

$B M$ transplantation studies suggest the cell-intrinsic function of $H u R$. To determine whether the hematopoietic progenitor cell phenotype in the Elavl1 ${ }^{\Delta / \Delta}$ mice is cell intrinsic, we conducted BM transplantation studies. Irradiated CD45.1 congenic recipients received BM cells from either Elavl1f/f or Elavl1f/fRosa26Cre ERT2 mice. At 10 weeks after transplantation, mice were treated with tamoxifen to delete Elavl1 in the hematopoietic compartment and were monitored over a period of 16 days. Interestingly, all of the recipient mice survived during the experimental time frame and did not exhibit symptoms of cachexia or weight loss, despite the fact that Elavl1 was efficiently deleted in the BM and the thymus (Figure $7 \mathrm{~B}$ ). Intestinal tissues of these chimeras appear normal (Figure 7C), suggesting that the effect of Elavl1 deletion on hematopoietic progenitor cells is cell intrinsic.

The immune organs were analyzed by FACS analysis 16 days after Elavl1 deletion. Total BM and thymocyte counts declined drastically in the mice with Elav1-deleted BM (Figure 7G). $B$ progenitors and pro- $B$ and immature $B$ cell numbers were strongly reduced whereas mature $B$ cells were not (Figure 7D). Likewise in the thymus, the double-positive population was significantly reduced (Figure $7 \mathrm{E}$ ), whereas the $\mathrm{CD}^{+}$and $\mathrm{CD}^{+}$ populations did not change. The apparent increase in the double-negative population is due to the loss of total cell numbers. In the myeloid cells of the BM, there was substantial decrease in total monocytes, whereas neutrophils were not significantly affected (Figure 7F). Interestingly, HuR expression is higher in monocytes than in polymorphonuclear leukocytes (PMNs) isolated from peritoneal exudates induced by thioglycollate elicitation (Supplemental Figure 7).

Loss of Elavl1 affects genes involved in hematopoiesis, apoptosis, and proliferation in BM. Given that HuR is a RNA-binding protein involved in posttranscriptional regulation, we searched for the gene expression changes in progenitor cells upon HuR loss. We conducted a microarray analysis of total RNA from BM of Elavl1 f/f and Elavl1 ${ }^{\Delta / \Delta}$ mice 24-48 hours after treatment with tamoxifen, which is the time frame before the occurrence of maximal cell death. As expected, the Elavl1 transcript was downregulated in BM cells of Elavl1 ${ }^{\Delta / \Delta}$ mice (60\%-90\% within 24-48 hours; Figure 8B).

Microarray data was analyzed by the Ingenuity pathway analysis (31). Of the 6,790 transcripts that exhibited significant changes $(P<0.001)$, genes classified to regulate cell death, cellular growth and differentiation, cell cycle, and organismal survival were most prominent (Figure 8A).

Hematopoietic transcription factors (Klf1, Tal1, Zfpm1, Gata1) were downregulated. In contrast, early HSC markers (Nanog, Sox2, Musashi [Msi1h and Msi2h]) were induced. This is consistent with loss of progenitors in multiple lineages (CLP, ELP, common myeloid progenitor), the resultant decline in differentiated cells, and a slight increase in HSCs. Regulators of apoptosis were altered by HuR loss. For example, survival factors (Bcl2, Bcl- $\mathrm{X}_{\mathrm{L}}$, survivin) were downregulated, whereas apoptotic inducers (c-Myc, caspase-9, caspase-8associated protein-2, NOXA, PUMA) were upregulated. Among the cell-cycle regulator transcripts, we noticed a reduction in cyclin E, proliferating cell nuclear antigen (PCNA), and the Ki67 antigen (MKi67). However, we also noticed an increase in the expression of mRNA for cyclin G1 (Ccng1), a target of the tumor suppressor p53. Interestingly, the $\mathrm{G}_{1} / \mathrm{S}$ cell-cycle regulator $\mathrm{p} 21^{\text {Cip } 1 / \text { Waf1 }}$, a major 

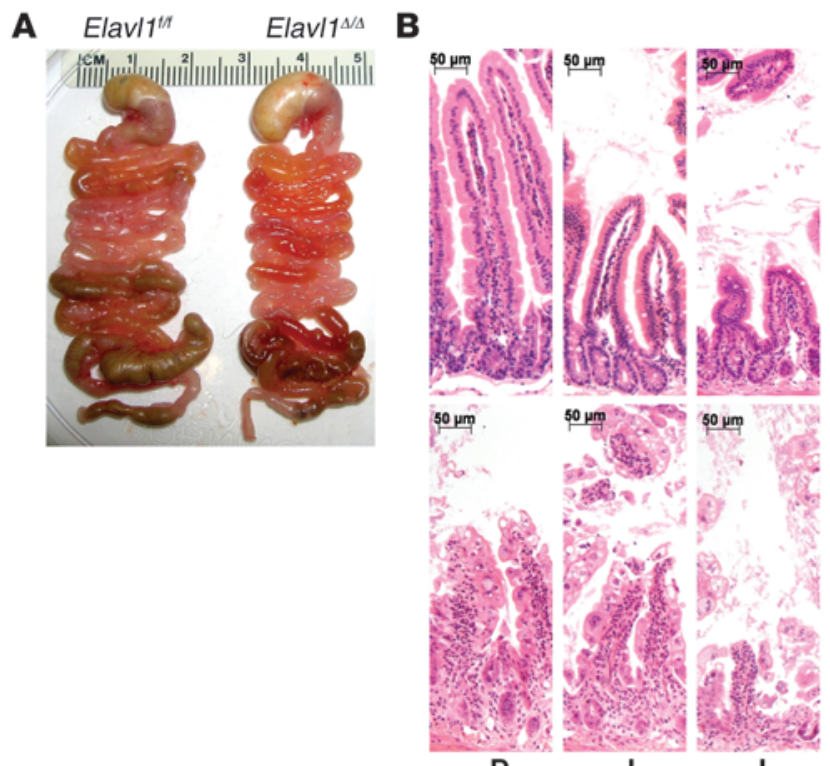

J

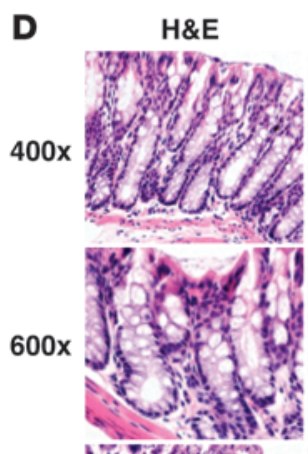

TUNEL
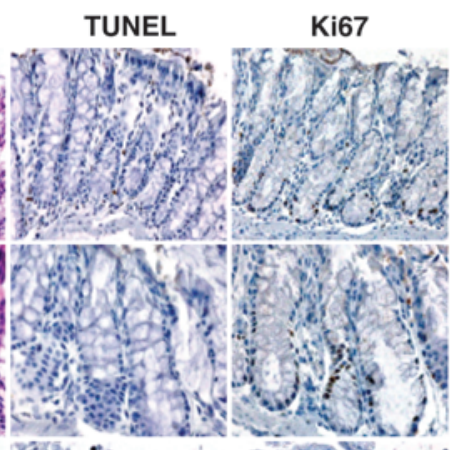

(5)
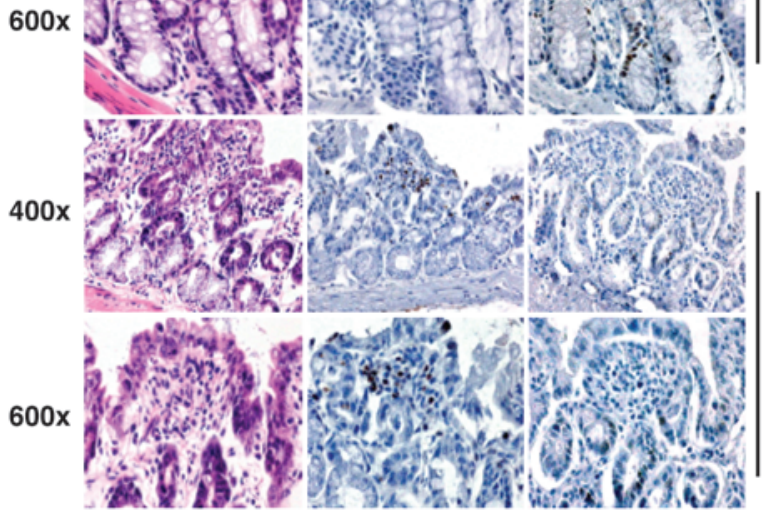

C
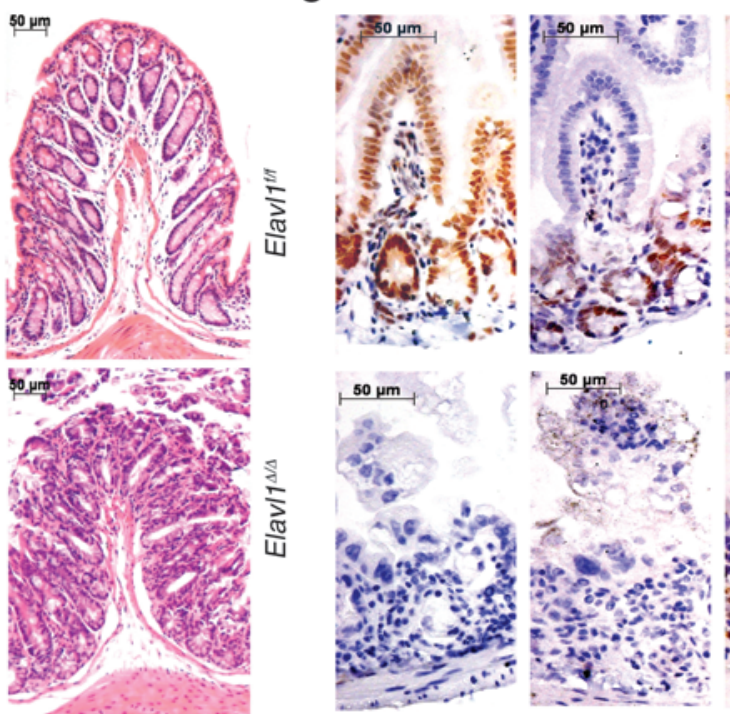

HuR
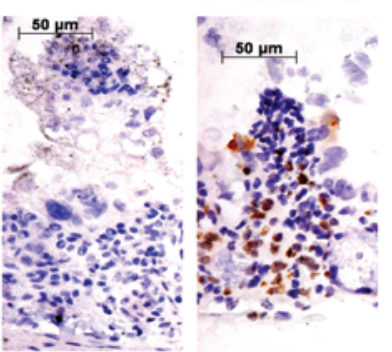

C

Ki67

TUNEL

E Time (h)

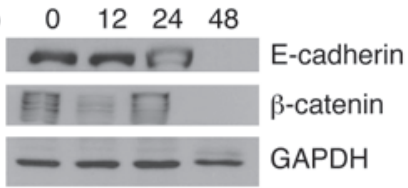

Elavl1 ${ }^{\text {th }}$

Elavl1 $1^{1 / 4}$

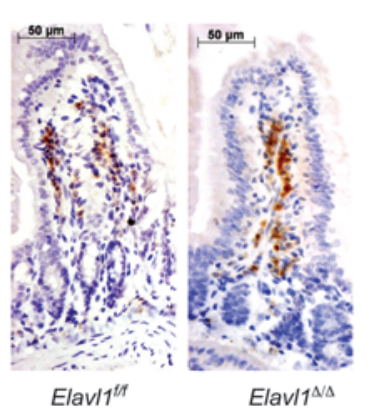

\section{Figure 5}

Role of HuR in intestinal integrity and progenitor cell survival. (A) Abnormal appearance of the GI tract 4 days after deletion of Elavl1. Note the distended stomach and thin-walled intestinal tract devoid of food. (B) H\&E stain of intestinal tissue sections from Elav/1/f/ and Elav/1 $1 / \Delta$ mice 4 days after tamoxifen administration. Note the effect of Elavl1 deletion on villus atrophy, epithelial cell disruption in the small intestine, and loss of tissue architecture and goblet cells in the colon. D, duodenum. Original magnification, $\times 20$. Scale bar: $50 \mu \mathrm{m}$. (C) Immunohistochemical staining of small intestinal tissue sections from Elav/1/ff and Elav/1 ${ }^{\Delta / \Delta}$ mice 48 hours after tamoxifen treatment with either HuR or Ki67 antibody or TUNEL stain. Note that although HuR is primarily expressed in the epithelial cells, some stromal cells in the lamina propria also show immunoreactivity. Original magnification, $\times 20$. Scale bar: $50 \mu \mathrm{m}$. (D) Immunohistochemical staining of colon sections from Elav/1/f/ and Elav/1 ${ }^{\mathrm{A} / \Delta}$ mice 48 hours after tamoxifen treatment with either H\&E stain, TUNEL, or Ki67 stain. Original magnification, $\times 400$ (first and third rows); $\times 600$ (second and fourth rows). (E) IB analysis of E-cadherin and $\beta$-catenin expression in intestinal tissue extracts isolated from Elav/1 ${ }^{\Delta / \Delta}$ mice upon tamoxifen treatment. (F) Immunohistochemical staining of F4/80 (myeloid marker) in small intestinal tissue sections from Elav/1 $1^{\text {t/f }}$ and Elav/1 ${ }^{\mathrm{s} / \Delta}$ mice 48 hours after tamoxifen treatment. Note that an intact villus was chosen to highlight the lamina propria and the epithelial compartments. Original magnification, $\times 20$. Scale bar: $50 \mu \mathrm{m}$.

p53-regulated inhibitor of cyclin-dependent kinases (32), was also induced. Indeed, several genes in the p53 pathway were regulated by HuR loss. For example, cell-cycle regulators (Pcna, Rb1, 14-3-3o $[S f n])$, cell survival factors (Bcl2), apoptotic inducers (DR4 [Apo2],
TRAILR-1 [Tnfrsf10A], Noxa, PUMA), regulators of cell-cycle arrest $\left(p 21^{\text {Cip1/Wafl } 1}, A t m\right)$ as well as the $p 53$ mRNA itself were induced upon the deletion of Elavl1 (Figure 8, B-D, and Supplemental Table 1), suggesting the involvement of the p53 pathway. 
A

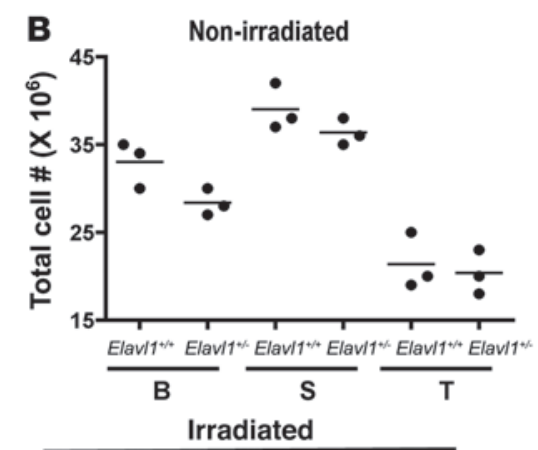

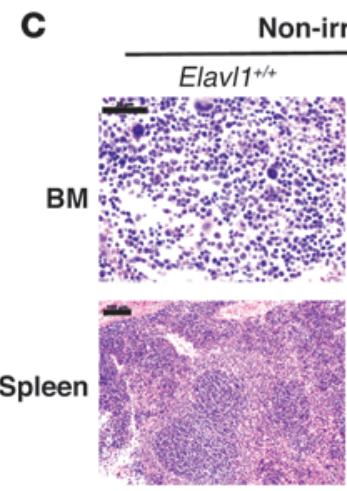

D

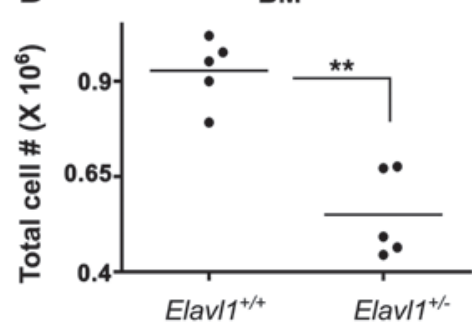

Analyzed

Elavl1 $1 \%$
Day 8<smiles>C1CCCCC1</smiles>
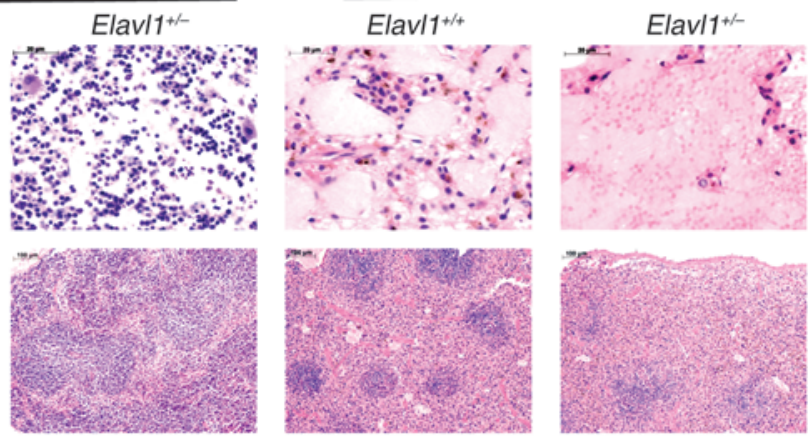

Spleen
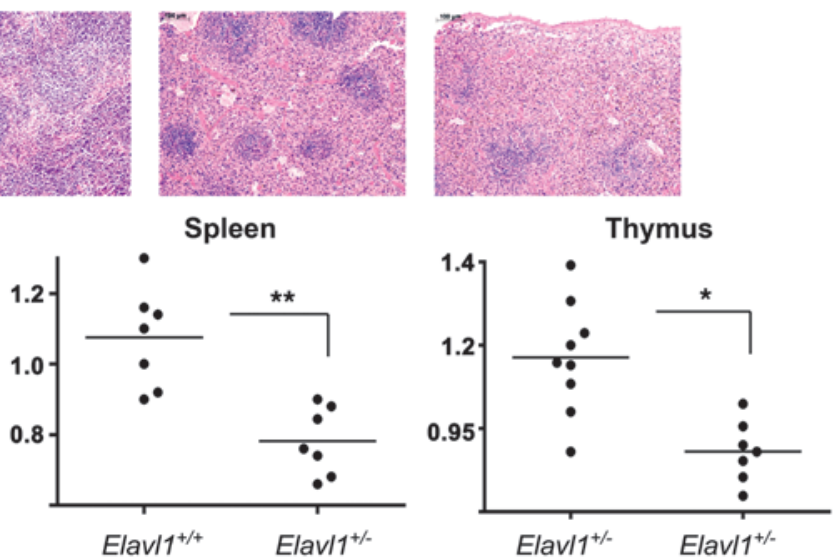

Figure 6

Elav/1+/- mice have increased sensitivity to whole-body irradiation. (A) A schematic of Elav/1+/+ and Elav/1+/- mice exposed to radiation. (B) Total cell number in BM, spleen, and thymus in nonirradiated Elav/1+/+ and Elavl1+/- mice $(n=3)$. (C) H\&E stain of bone and spleen tissue sections from Elav/1+/+ and Elav/1+/- mice before and after irradiation. Original magnification, $\times 10$ (spleen); $\times 40$ (BM). Scale bar: $20 \mu \mathrm{m}$ (BM); $100 \mu \mathrm{m}$ (spleen). (D) Total number of cells isolated from BM, spleen, and thymus of irradiated Elav/1+/+ and Elav/1+/- mice $(n \geq 5)$. Horizontal bars indicate mean of data points, and each dot represents an analyzed mouse (B and $\mathbf{D})$.

Loss of Elavl1 leads to p53 induction in hematopoietic and intestinal progenitor cell compartments. To determine whether the p53 pathway is induced in a differentiation-specific manner in the BM cells, we examined the expression of $\mathrm{p} 53$, its upstream regulator $\mathrm{Mdm}-2$, and the downstream gene $p 21^{\text {Cip } 1 / \text { Wafl }}(32)$ in sorted BM populations (Figure 9A). The induction of the p53 mRNA was observed in both the quiescent HSCs as well as the proliferating ELP populations. This was correlated with the reduction in $\mathrm{Mdm}-2$ expression in both HSC and ELP populations, suggesting that loss of $\mathrm{Mdm}-2$ led to the induction of $\mathrm{p} 53$. Interestingly, $\mathrm{p} 21^{\mathrm{Cip} 1 / \mathrm{Waf1}}$ was induced strongly only in the ELP population, whereas c-myc, an important proapoptotic factor, was induced in both HSC and ELP populations.

IB analysis of BM extracts from Elavl $1^{\Delta / \Delta}$ mice indicated that $\mathrm{p} 53$, p21 Cip1/Waf1, Bax, p19ARF, and c-myc levels were induced as HuR levels declined (Figure 9B). Phospho-CHK2 was induced, whereas $\mathrm{Mdm}-2$ and Bcl-2 levels declined. These data are consistent with the induction of p53-dependent apoptotic response.

In the intestine, the deletion of Elavl1 and induction of p53 and

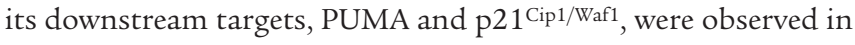

progenitor cells of the crypts (Figure 9C). Expression of Musashi, a marker for early intestinal stem cells (33) was not reduced, suggesting that early intestinal stem cells do not undergo apoptosis upon Elavl1 deletion. In contrast, proliferation (Ki67 positivity) was dramatically reduced and TUNEL positivity was induced in small intestinal crypts, suggesting that progenitors (also known as transit amplifying cells) undergo cell-cycle arrest and apoptosis upon HuR loss. p53 and its downstream target p21 Cip1/Waf1 were induced specifically in the intestine but not in lung and liver of Elavl1 ${ }^{\Delta / \Delta}$ mice (Figure 9D), suggesting that HuR regulation of the p53 pathway is specific for progenitor cells.

$H u R$ binds to $M d m-2$ and regulates $p 53$ levels in vitro. Next, we examined the effect of HuR loss on mouse embryonic fibroblasts (MEFs) derived from Elavl1f/f and Elavl1f/fRosa26Cre ${ }^{E R T 2}$ mice. The 4-hydroxytamoxifen (4HT) treatment suppressed HuR expression and cell proliferation, while inducing $\mathrm{p} 53$ and $\mathrm{p} 21^{\text {Cip } 1 / \text { Waf1 }}$ expression (Figure 10, A and B). Mdm-2 was downregulated at early time points, while p19AR, an inhibitor of $\mathrm{Mdm}-2$ function, was induced. Loss of Mdm-2 expression and the inhibition of its 

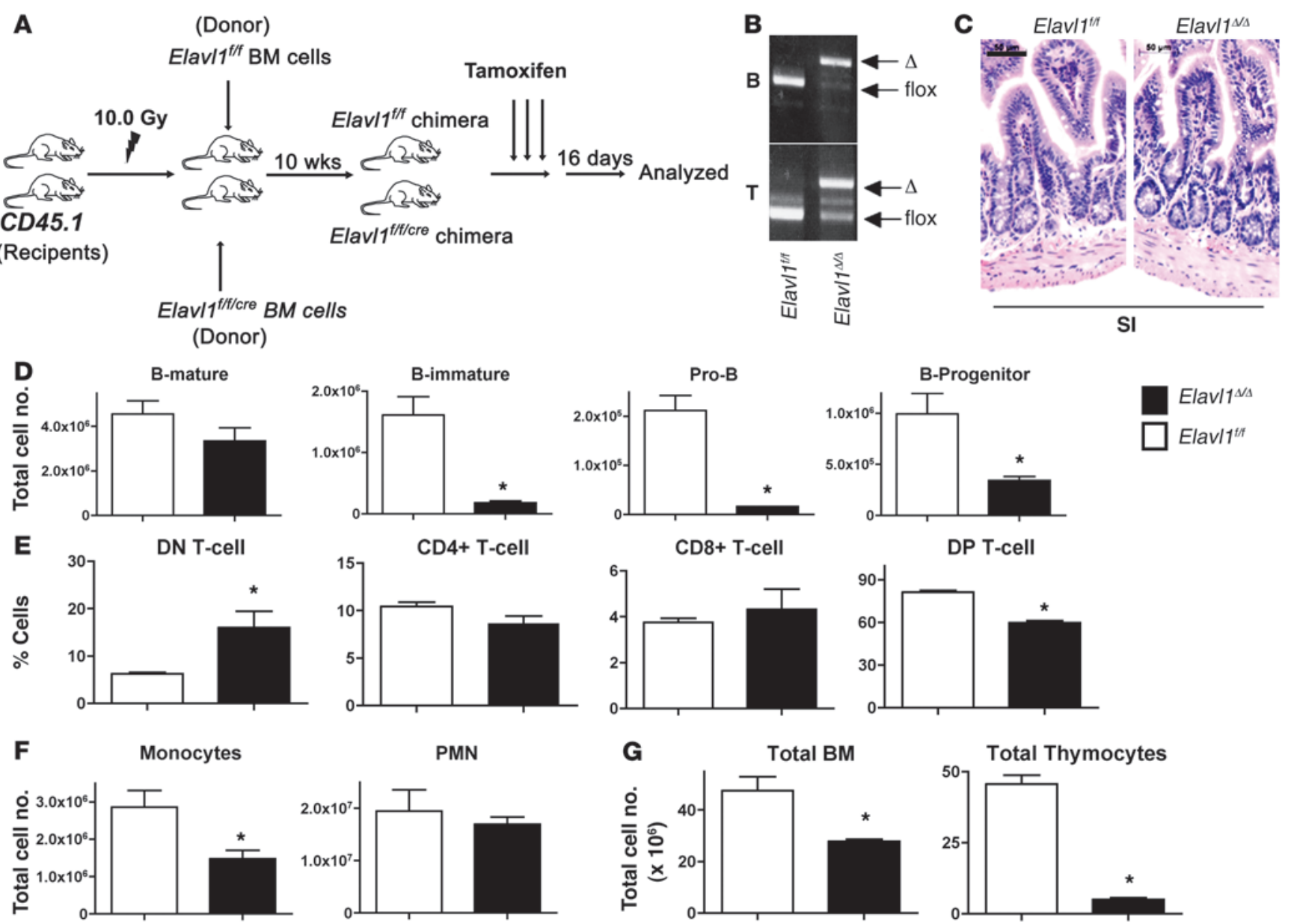

Figure 7

Cell-intrinsic effect of HuR. (A) A schematic of BM transplant. (B) PCR analysis of genomic DNA from indicated organs of mice 3 days after tamoxifen administration. (C) H\&E stain of small intestinal tissue sections from Elav/1/f/f and Elav/1 $1 / \Delta$ chimera at day 16 . Original magnification, $\times 20$. Scale bar: $50 \mu \mathrm{m}$. (D-F) Isolated cells from BM (D and F) and thymus (E) were analyzed by FACS as described. Cell numbers of different

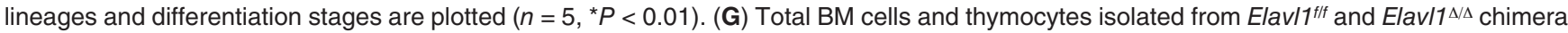
on day 16 are shown $\left(n=5 ;{ }^{*} P<0.01\right)$.

activity by $\mathrm{p} 19^{\mathrm{ARF}}$ may be responsible for the induction of $\mathrm{p} 53$ in MEFs. Interestingly, c-myc, which activates p19ARF (34), was substantially upregulated in a time-dependent manner. Furthermore, phosphorylation of $\mathrm{CHK} 2$, a check point kinase that is known to activate p53 activity upon DNA damage (32), was induced, suggesting that HuR loss induces cell stress response. However, CHK2 protein level was not altered, suggesting that it is activated by an upstream kinase. Indeed, ATM, a DNA damage-inducible kinase, was strongly induced in the BM upon HuR loss (Figure 8D).

$M d m 2$ mRNA and protein levels were rapidly downregulated upon $\mathrm{HuR}$ loss, suggesting that $\mathrm{HuR}$ may directly regulate the expression of $\mathrm{Mdm}-2$ at the posttranscriptional level. Indeed, the 3 '-untranslated region of the $M d m 2$ mRNA is AU rich and contains several copies of AREs and canonical HuR-binding motifs (Supplemental Figure 9). We tested whether HuR binds to Mdm2 mRNA by immunoprecipitating MEF cell extracts with anti-HuR or control IgG and analyzing the presence of Mdm2 transcript by quantitative real-time PCR (q-RT-PCR). As shown in Figure 10C, Mdm2 mRNA was recovered from HuR immunoprecipitates in a dose-dependent manner. In addition, loss of HuR led to reduction in Mdm2 mRNA half-life from 1.3 to 0.8 hours, suggesting that HuR regulates the stability of $M d m 2$ mRNA (Figure $10 \mathrm{D})$. To determine whether regulation of $\mathrm{Mdm}-2 / \mathrm{p} 53$ pathway is essential for cell death, we treated MEFs with $4 \mathrm{HT}$, followed by transfection with Mdm-2 expression plasmid (Figure 10E), and quantified cell proliferation. Indeed Mdm-2 overexpression rescued suppression of cell proliferation induced by HuR loss (Figure 10F).

\section{Discussion}

Several RNA-binding proteins that interact with the AREs in the 3'untranslated region of transcripts have been characterized in vitro and in vivo $(35,36)$. These proteins regulate RNA stability, transport, and translation. HuR is unique in that it stabilizes the transcripts involved in inflammation such as cyclooxygenase- 2 and tumor necrosis factor- $\alpha(8,9,36,37)$. However, its function is not limited to the regulation of genes involved in the inflammatory response ( 5 , $7,38)$. Indeed, recent studies suggest that HuR may regulate gene expression by modulating the activity of miRNAs $(4,39)$.

The interaction of HuR with transcripts involved in various cellular processes suggests its multifunctionality. Indeed, HuR function has been implicated in numerous biological processes includ- 
A
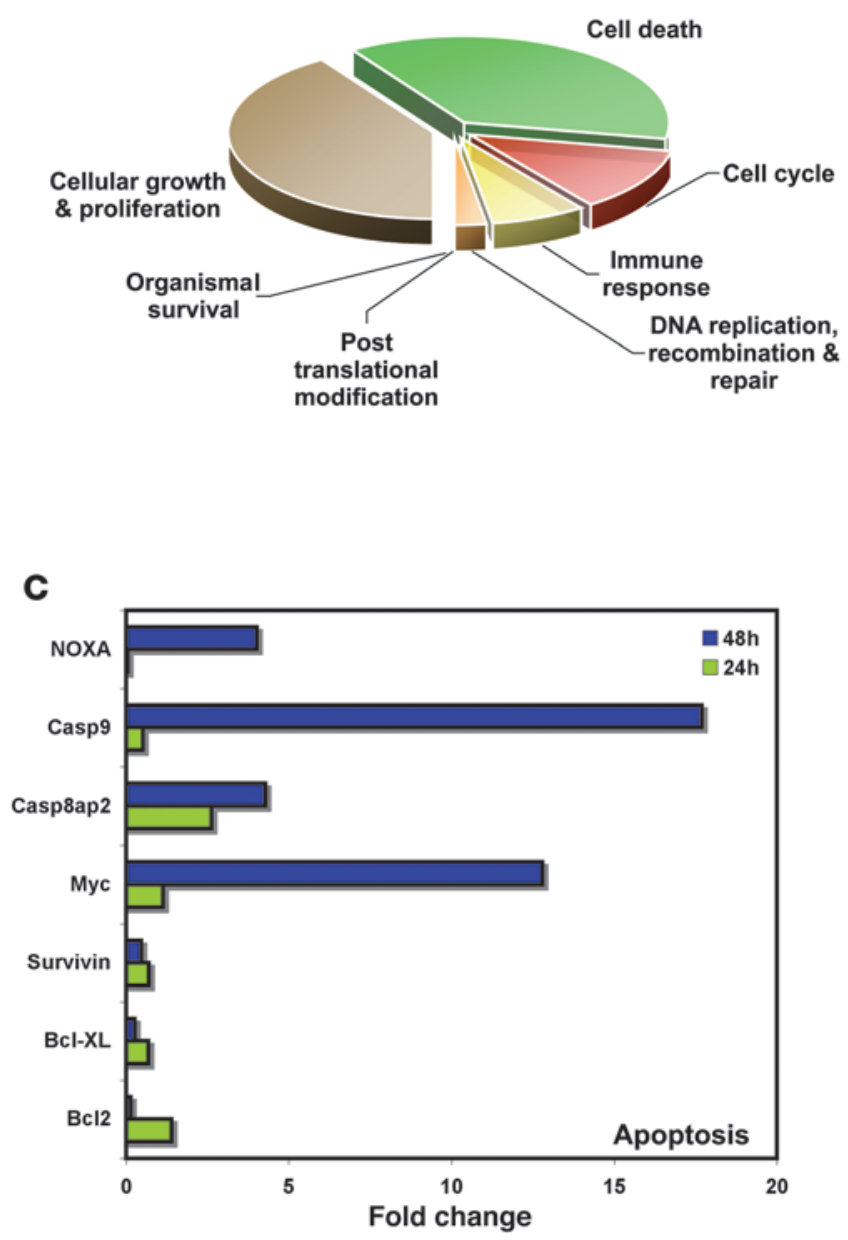

B

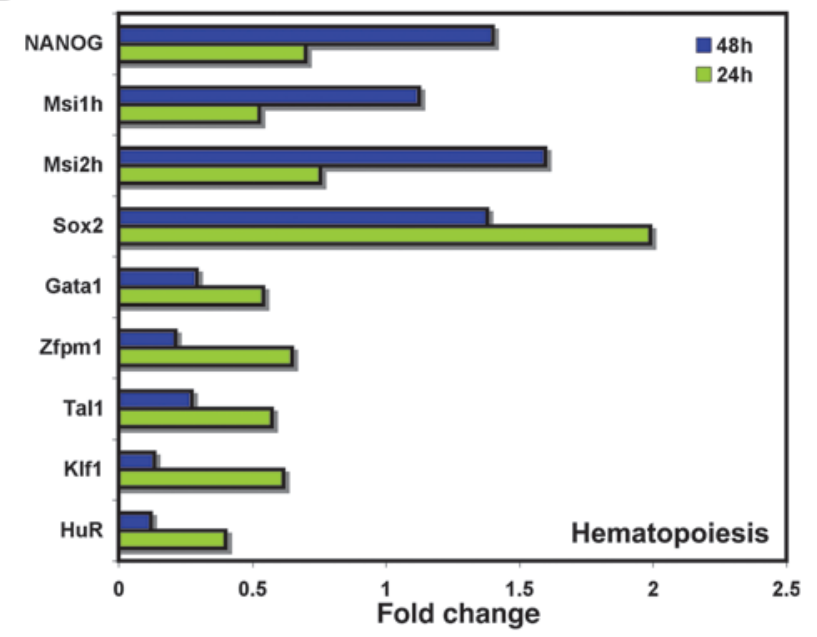

D

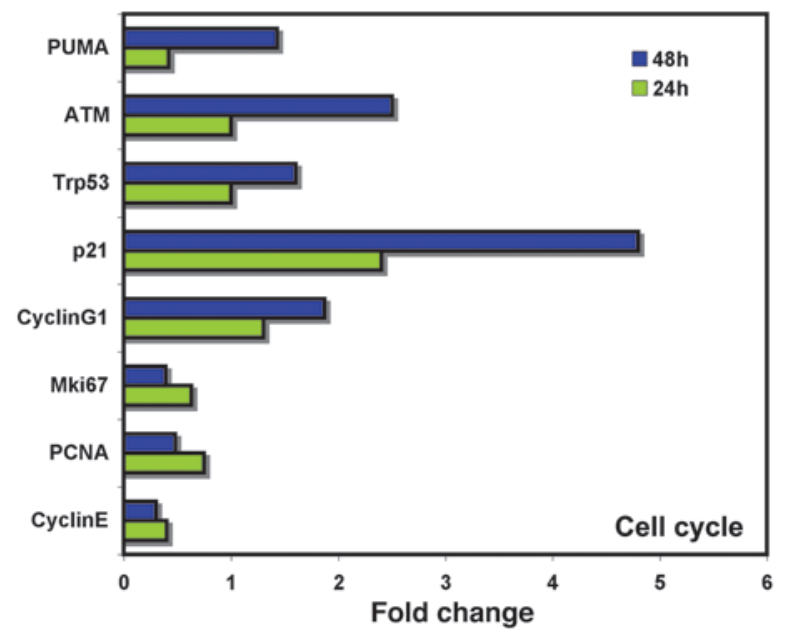

Figure 8

Microarray analysis of the transcript expression pattern in BM upon loss of HuR. (A) Ingenuity pathway analysis (31) was used to classify the transcriptome changes that occur in BM after Elavl1 deletion. (B-D) Changes in specific genes involved in hematopoiesis (B), apoptosis (C), and cell-cycle control (D) after Elavl1 deletion. Graphs represent Elav/1 ${ }^{\Delta / \Delta / E l a v / 1 / f ;} ; n=3$; (transcripts shown have $P<0.001$; for downregulated transcripts, $x$ axis represents $-1 / x$-fold change in gene expression).

ing cell proliferation, survival, angiogenesis and cancer in addition to the aforementioned function in inflammation $(5,7,38)$. However, in vivo functions of HuR are not well understood, especially in the postnatal period. A recent paper described the embryonic lethal phenotype of Elav1 $1^{-/-}$mice and suggested that HuR regulates placental development (18). Thus, although an essential role of HuR in embryonic development was described, its function in the adult organisms is not understood.

Our inducible global gene deletion model indicated that HuR plays a fundamental role in normal organismal survival and homeostasis. As HuR levels declined, rapidly proliferating tissues were affected first. Indeed, tissues with high cell proliferation and turnover express the highest levels of HuR in vivo. Thus HuR may be upregulated during periods of active cell proliferation, in which it plays an essential role in cell survival and is downregulated in more differentiated cells. Indeed, expression and subcellular localization of HuR shows a dynamic pattern during hematopoiesis. Our study, however, did not examine the functions of HuR in differentiated cells. The mouse model we developed will be useful in this purpose for delineating the tissue specific function of HuR.

The effect of HuR loss in hematopoietic and intestinal systems is profound and rivals lethal radiation exposure. This underscores the importance of posttranscriptional gene regulatory mechanisms in progenitor cells during periods of rapid cell expansion. At the cellular level, HuR function is needed for cell survival; lack of HuR led to rapid induction of apoptosis. Differentiated cells and quiescent stem cells do not require HuR for survival. BM transplantation studies indicate that the requirement for $\mathrm{HuR}$ in progenitor cell phenotype is cell intrinsic and not driven by generalized organismal demise.

Microarray studies in BM cells indicated that gene expression changes accompanied cellular alterations. For example, regulators of hematopoiesis were downregulated, cell-cycle promoters were suppressed and inhibitors induced, and apoptotic inducers were increased. Interestingly, the p53 pathway was induced as 


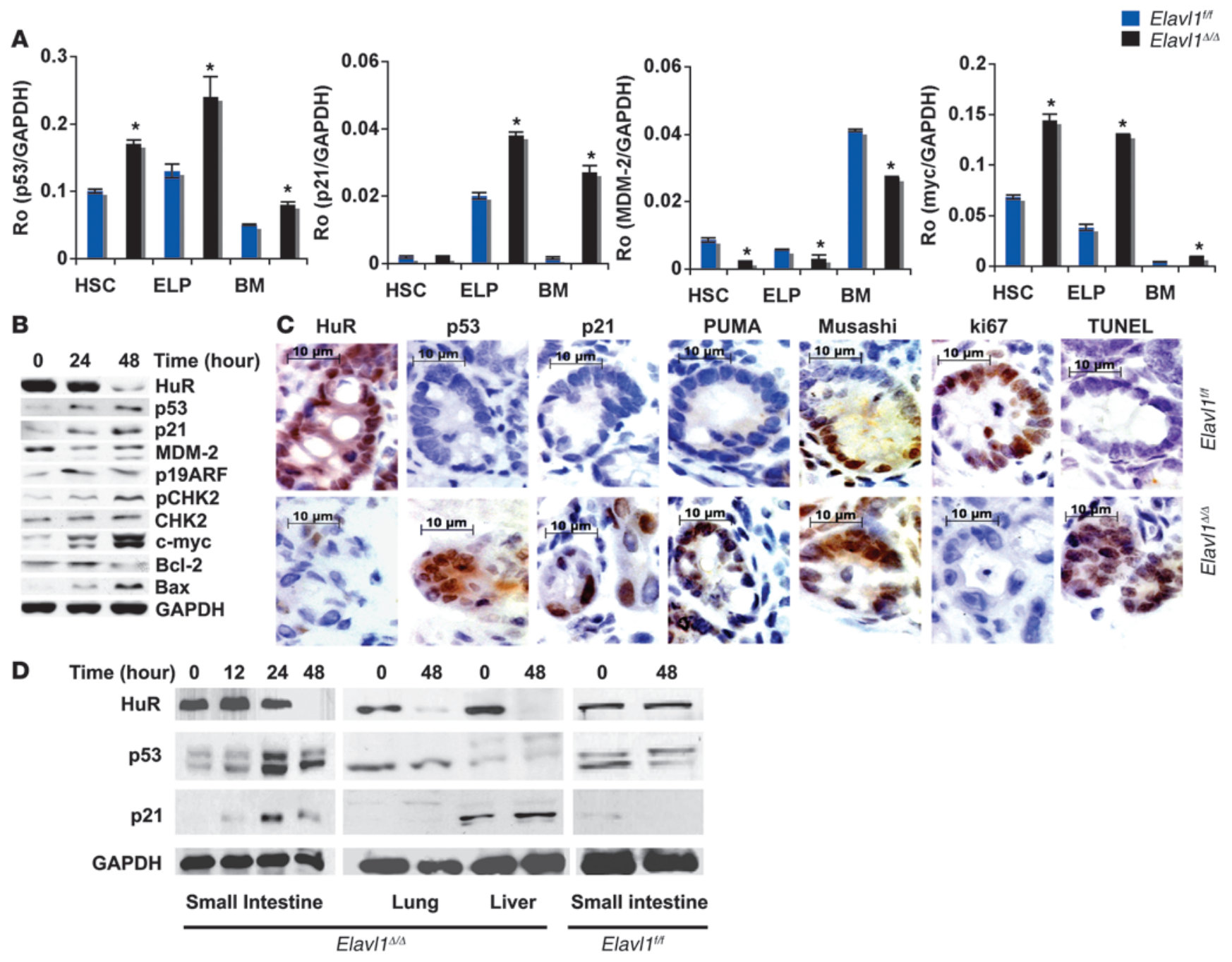

Figure 9

Induction of the p53 pathway in hematopoietic and intestinal progenitors with loss of HuR. (A) Total BM cells isolated from Elavl1/f/ and Elav/1 $1 / \Delta$ mice $(n=3) 48$ hours after tamoxifen treatment were pooled and sorted by FACS to obtain HSCs and ELPs. Total RNA was isolated, and gene expression for $p 53, p 21^{\text {Cip } 1 / \text { Waf1 }}, M d m 2$, and $c$-myc mRNA levels was determined by q-RT-PCR $\left({ }^{*} P<0.05\right)$. (B) IB analysis of BM extracts from Elav/1 $\Delta / \Delta$ mice treated with tamoxifen for the indicated time interval. (C) Immunohistochemical staining of intestinal crypts from Elav/1/ff and Elav/1 $1 / \Delta$ mice at 48 hours after tamoxifen administration. Original magnification, $\times 40$. Scale bar: $10 \mu \mathrm{m}$. (D) IB analysis of tissue extracts isolated from Elav/1/ff and Elav/1 ${ }^{\Delta / \Delta}$ mice after tamoxifen treatment.

HuR levels declined. Moreover, we uncovered what we believe to be a novel regulation of $\mathrm{Mdm}-2$, an important E3 ubiquitin ligase for p53. HuR binds and stabilizes the Mdm2 mRNA. Indeed, Mdm-2 expression was rapidly downregulated in progenitor cells as HuR levels declined. Interestingly, c-myc, which is repressed by HuR through recruitment of the miRNA let-7 (39) was strongly induced in cells that lack HuR. It is known that c-myc activates p19ARF (34). Thus, combined effects of Mdm-2 expression and the inhibition of its activity by $\mathrm{p} 19^{\mathrm{ARF}}$ may be responsible for the strong induction of p53. Interestingly, CHK2 phosphorylation and ATM induction were seen, suggesting the activation of the cell stress response pathway in the absence of $\mathrm{HuR}$. The ability of Mdm-2 expression to restore cell proliferation in MEFs that lack HuR strongly suggests that activation of the p53 pathway is essential in the progenitor cell apoptosis phenotype. These data imply that HuR regulated posttranscrip- tional mechanisms are required to keep p53 levels in check in proliferating progenitor cells.

In conclusion, our studies uncovered what we believe to be a novel posttranscriptional gene regulatory system in progenitor cells that requires HuR to regulate p53 expression. Since the HuR loss in this inducible gene deletion animal model phenocopies the radiation syndrome, HuR regulation of cell stress response in progenitor cells may be exploited in cancer therapy. For example, inhibition of HuR expression or function in cancer stem/progenitor cells may lead to rapid apoptosis and tumor shrinkage. In addition, preservation of HuR expression in normal cells may minimize the side effects of cytotoxic anticancer therapies, such as pancytopenia and radiation syndrome. As a corollary, increased HuR function in progenitor cells may enhance the efficacy of stem cell transplant therapy. Further studies should examine the role of HuR in embryonic and inducible pluripotent stem cells. 
A

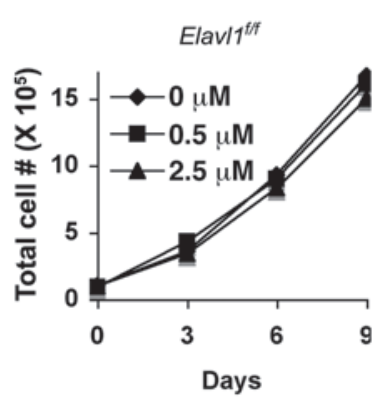

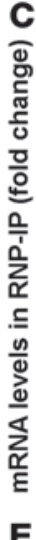

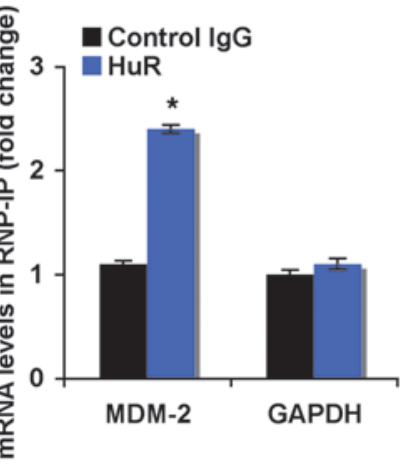

$\mathbf{E}$
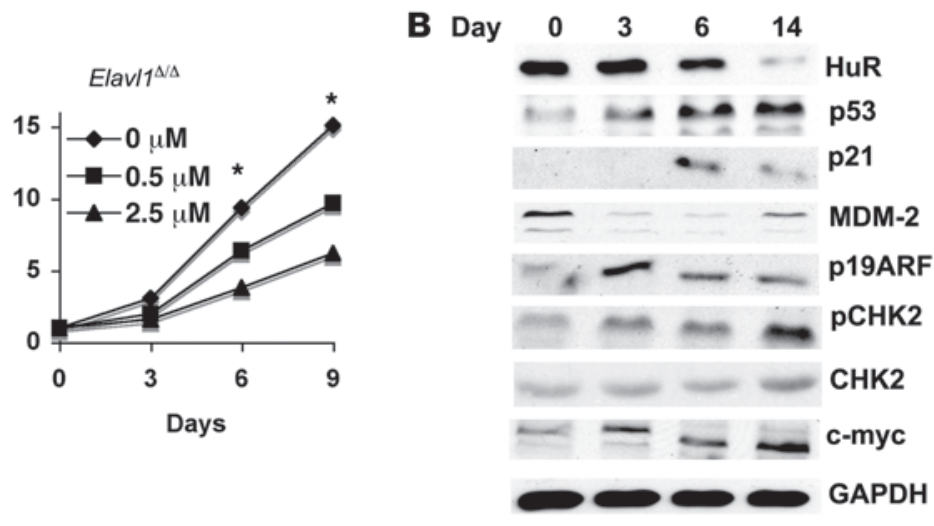

D

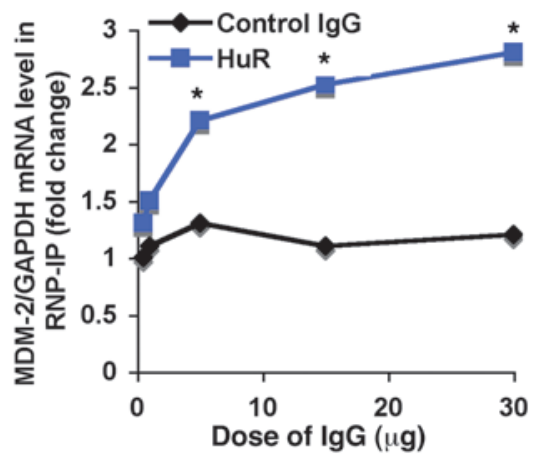

$\mathbf{F}$

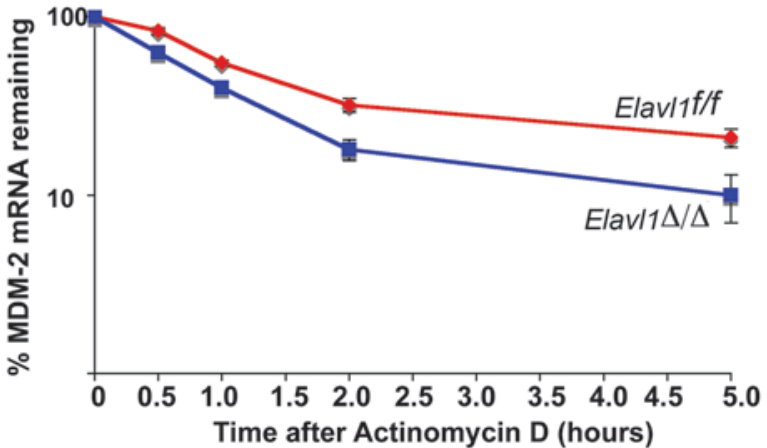

Time after Actinomycin D (hours)

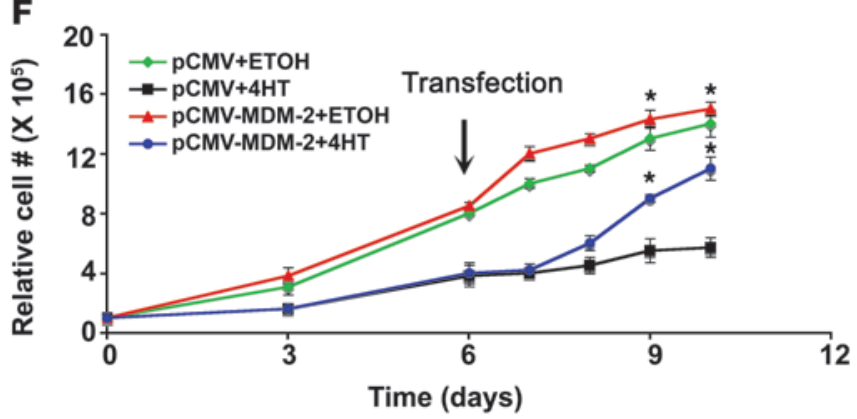

Figure 10

Binding and regulation of Mdm-2 by HuR in MEFs. (A) Growth curve and (B) IB analysis of MEF cells isolated from Elav/1/f/ and Elav/1/f/RosaCre mice treated with different doses of $4 \mathrm{HT}$ for the indicated time periods $\left({ }^{*} P<0.05 ; n=3\right)$. For IB analysis, MEFs treated with $2.5 \mu \mathrm{M}$ of $4 \mathrm{HT}$ are shown. (C) Ribonucleoprotein (RNP) complexes were immunoprecipitated from MEFs, using different doses of HuR-specific antibody or mouse IgG, and immunoprecipitated RNA was purified and analyzed for the level of Mdm2 transcript by q-RT-PCR. $\left({ }^{*} P<0.05 ; n=2\right)$. (D) Percentage of Mdm2 mRNA remaining was determined in Elav/1 $1^{t / f}$ and Elav/1 ${ }^{\Delta / \Delta}$ MEFs upon addition of Actinomycin $D$ for the indicated time interval $(n=2)$. $(E)$ IB analysis of Mdm-2 overexpression in MEFs transfected with pCMV6-Mdm-2 cDNA. (F) Growth curve analysis of Elav/1f///cre MEFs transfected with pCMV6-Mdm-2 or pCMV6 alone, in presence of $4 \mathrm{HT}$ or vehicle (ethanol, ETOH) for the indicated time intervals $\left({ }^{*} P<0.05 ; n=3\right.$ ).

\section{Methods}

Mice. Animal procedures were approved by the Institutional Animal Care and Use Committee of the University of Connecticut Health Center. Tail DNA samples were used for PCR to monitor the deletion of HuR exons 2-6 and the presence of the Cre allele. Rosa26Cre/ER ${ }^{\mathrm{T} 2}$ mice were provided by G.-H. Fong (University of Connecticut Health Center; ref. 20). Tamoxifen (Sigma-Aldrich) was administered to 6- to 8-week-old Elavl1f/f and Elavl1ffRosa26Cre/ERT2 mice by gavage at the dose of $200 \mathrm{mg} / \mathrm{kg}$ in corn oil. In most experiments, tamoxifen was given daily for 3 consecutive days.

Mice were injected with $200 \mu \mathrm{l} \mathrm{BrdU}$ solution $(5 \mathrm{mg} / \mathrm{ml}$ ) (SigmaAldrich) in PBS intraperitoneally for 4 hours, and the tissues were harvested for immunohistochemistry with the anti-BrdU antibody. Survival curves were generated using the Kaplan-Meier method, and significance was evaluated using the log-rank test.

Immunohistochemistry. Immunohistochemical staining was conducted in paraffin-embedded sections, according to the manufacturer's instructions, with the following antibodies: biotinylated HuR monoclonal antibody (1:200) (5), rat monoclonal Ki67 (1:200, Dako), mouse monoclonal p53 (1:150, Santa Cruz Biotechnology Inc.), rabbit polyclonal PUMA (1:200, Cell Signaling Technology), and IgG with a Vectastain ABC kit (Vector Laboratories).

TUNEL staining was performed using an ApopTag Peroxidase In Situ Apoptosis Detection Kit (Chemicon International) according to manufacturer's instructions. The apoptotic index was scored in full longitudinal 
sections of crypts and villi and lamina propria as the number of positive apoptotic cells normalized to total number of cells present in crypts and villi and lamina propria, respectively.

Quantitative RT-PCR. Total RNA was purified and q-RT-PCR was done using an ABI 7900HT instrument (Applied Biosystems) as described (40).

Microarray analysis. Illumina Mouse- 6 microarray was performed (Translational Genomics core, University of Connecticut Health Center) with the total RNA sample isolated from BM of Elavl1/f/f and Elavl1 ${ }^{\Delta / \Delta}$ mice $(n=3)$. The chips contained approximately 48,000 transcripts from the mouse sequence-verified NIA $15 \mathrm{k}$ cDNA library plus the NIA 7.4k cDNA clone set (http://gsun.grc.nia.nih.gov/cDNA/cDNA.html). Spot intensities were analyzed from scanned images using Scan Array Express (Perkin Elmer). Data were analyzed through the use of Ingenuity Pathways Analysis software suite (Ingenuity Systems).

Protein analysis. For IB analysis, protein extracts from murine tissues or cells were obtained by extraction in RIPA lysis buffer and processed as described (40).

Flow cytometry. All conjugated antibodies and secondary reagents used for flow cytometry were purchased from BD Biosciences or eBiosciences.

For analyses of early hematopoietic and lymphoid progenitors, samples were initially incubated with a cocktail of biotinylated antibodies against multiple mature hematopoietic lineage (Lin) markers, including CD3/CD4/CD8 (T lymphocytes), CD45R/B220 (B lymphocytes), CD11b (monocytes and macrophages), F4/80 (mature macrophages), Gr-1 (granulocytes), Ter119 (erythroid progenitors), and NK1.1 (natural killer cells). The samples were incubated at $4^{\circ} \mathrm{C}$ and then washed with staining media. As a second stage, samples were further incubated with streptavidin coupled to PECy7, Sca-1 coupled to FITC, CD117 coupled to allophycocyanin (APC), and CD127 coupled to PE. These 3 last antibodies label hematopoietic progenitors in the context of lineage-negative $\left(\mathrm{Lin}^{-}\right)$populations.

For analysis of B cell lineage development, samples were initially incubated with a biotinylated antibody against IgM, followed by a second step with PECy7-conjugated streptavidin, FITC-conjugated CD45R/B220, PEconjugated CD19, and APC-conjugated CD117. For myeloid development, cell suspensions were stained with biotinylated anti-CD115 followed by a second step with PE-conjugated streptavidin, APC-conjugated CD11b, and FITC-conjugated Gr-1. For erythroid progenitor analysis, cell suspensions were stained with PE-conjugated Ter119 and, in certain experiments, together with APC-conjugated CD117.

Analysis of thymocytes was done by staining cell suspensions with antiCD3 coupled to FITC, CD4 coupled to PE, and CD8 coupled to APC. In all the above described staining protocols, cells were suspended in a solution of staining media containing $1 \mathrm{mg} / \mathrm{ml}$ of propidium iodide (PI), and analyses were done after gating out dead cells.

For apoptosis, Vybrant Apoptosis assay kit containing Alexa Fluor 488 annexinV/PI was used according to manufacturer's instruction. The cells were counterstained either with biotinylated anti-IgM, followed by PECy7-conjugated streptavidin and PE-conjugated anti-CD45R/B220 or CD4 coupled to PE plus CD8 coupled to APC. After staining, cells were resuspended in staining media containing PI. For analyses, cells positive for Annexin V and negative for PI were considered apoptotic, while cells double positive for PI and Annexin V were considered necrotic.

For cell proliferation assay, 4 hours after mice were injected with BrdU, cells were isolated from organs and stained initially with biotinylated anti-IgM followed by PE-conjugated streptavidin plus APC-conjugated CD45R/B220. Cells were then fixed in $95 \%$ ethanol, permeabilized in $1 \%$ paraformaldehyde $/ 0.01 \%$ Tween-20, and treated with DNAse-I (Sigma-Aldrich). Finally, cells were stained with FITC-conjugated anti-BrdU antibody.

All analyses were done in a BD FACSCalibur flow cytometer, and data was processed using Cell Quest analysis package software.
Cell sorting. For sorting hematopoietic progenitors samples were first stained with biotinylated lineage cocktail followed by PECy7-conjugated streptavidin plus FITC-conjugated anti-Sca1, APC-conjugated CD117Alexa Fluor 750, APC-conjugated CD127, and PE-conjugated CD135. LT-HSCs, ST-HSCs, and ELPs were sorted (41). For isolating B cells, BM suspensions were stained with biotinylated anti-IgM followed by APCand Cy5.5-conjugated streptavidin plus FITC-conjugated CD45R/B220. Immature B cells (pre-pro, pro, and pre B cells) were sorted according to the phenotype $\mathrm{CD} 45 \mathrm{R} / \mathrm{B} 220^{+} \mathrm{IgM}^{-}$, while more mature cells were defined as $\mathrm{CD} 45 \mathrm{R} / \mathrm{B}_{22} 0^{+} \mathrm{IgM}^{+}$. All sorts were done in a BD FACS Aria equipped with 4 lasers and operated using the FACS Diva 6.0 software.

CFC assay. BM hematopoietic progenitor activity was evaluated as described (42).

Immunofluorescence. For immunostaining of cell-sorted population from BM, cells were cytospun, fixed, and stained with mouse monoclonal HuR antibody. Nucleic acid dye, TOPRO-3 (Molecular Probes) was used for nuclear staining. Slides were examined using a Zeiss LSM 510 Meta confocal microscope.

Generation and analysis of BM chimeras. Male CD45.1 recipient mice were irradiated with 2 doses of $5.0 \mathrm{~Gy}, 4$ hours apart, and immediately reconstituted intravenously, via the tail vein, with CD45.2 BM cells from either Elavl1 f/f or Elavl1 ff/Rosa-Cre-ERT2 mice. Chimerism was approximately $90 \%-96 \%$, at 10 weeks after transplant. Recipient mice were given tamoxifen at 10 weeks after transplant for 3 days and monitored over a period of 16 days. Single cell suspensions of BM, thymus, and peripheral blood were prepared and stained with the following antibodies: Alexa 700 anti-CD19, APC-conjugated anti-B220, FITC -conjugated anti-IgM, PE -conjugated anti-CD117, PacificBlue anti-CD11b, APC-conjugated Alexa 750 or APCconjugated anti-CD3, PE-conjugated anti-CD4, APC-conjugated anti-CD8, FITC-conjugated anti-Gr1, streptavidin anti-CD115, and APC-conjugated anti-CD45.2. Data gated on the CD45.2-positive fraction were acquired on an LSRII (BD) and analyzed using FlowJo (Tree Star).

Isolation and growth of MEFs. MEFs were isolated from intercrosses of Elavl1/f/f or Elavl1 ff/fRosa-Cre-ERT2 mice at day 14 and cultured as described (43). For 4HT treatment, MEFs were grown in DMEM/MEM/10\% FBS/1\% antibiotics in presence of different doses of $4 \mathrm{HT}$ or ethanol as vehicle for different time intervals. 4HT was added fresh each day over a period of 14 days. For growth curve, cells were washed, trypsinized, and counted in a Coulter counter. For the transfection experiment with MEFs, cells were grown in presence of $2.5 \mu \mathrm{M} 4 \mathrm{HT}$ or vehicle for 6 days, followed by transfection with either empty vector (pCMV6) or MDM-2 overexpression plasmid (pCMV6$\mathrm{Mdm}-2$, Origene, $1 \mu \mathrm{g}$ of DNA) with Fugene, according to manufacturer's instruction (Roche), and relative cell number was measured after 24, 48, 72 and 96 hours of transfection.

Mdm2 mRNA stability assay. MEFs from Elavl1f/f or Elavl1ff/Rosa-Cre-ERT2 were grown in presence of $2.5 \mu \mathrm{M} 4 \mathrm{HT}$ for 6 days. On day $6,10 \mu \mathrm{g} / \mathrm{ml}$ of Actinomycin D (Sigma-Aldrich) was added, and total RNA was isolated at various time intervals and the Mdm2 mRNA level normalized to GAPDH was measured by q-RT-PCR.

Isolation of monocytes and PMNs. Total numbers of monocytes or PMNs were measured in peritoneal lavage fluid after 4 days or 4 hours of intraperitoneal injection of thioglycollate broth (3\%), respectively.

Cell-cycle analysis. Total BMcells were isolated from Elavl1f/f or Elavl1 1f/Rosa-Cre-ERT2 mice fed with tamoxifen for 2 days. Total BM cells were sorted for progenitor population, fixed in ethanol and stained with PI in RNAse A buffer, and analyzed by flow cytometry. The distribution of cells between different phases of the cell cycle was determined by Modfit LT software.

Messenger ribonucleoprotein immunoprecipitation. Immunoprecipitation of endogenous Elavl1 mRNA complexes used to assess the association of the endogenous level of HuR with the endogenous level of Mdm2 mRNA was performed as described previously (44). 
Statistics. All data were expressed as mean \pm SEM. Paired 2-tailed Student's $t$ test was applied to determine statistical significance between 2 groups. $P$ values of less than 0.05 were considered statistically significant.

\section{Acknowledgments}

This work is supported by NIH grants HL49094 and CA-77839 to T. Hla. We thank Brent Graveley, Kamal Khanna, Shobha Thangada, Robert Clark, Elizabeth Wohlfert, and Guo-Hua Fong as well as Anupinder Kaur and Katie Lamothe for technical help and Gordon Carmichael, S.K. Dey, and Timothy F. Lane for critical comments.

1. Moore, M.J. 2005. From birth to death: the complex lives of eukaryotic mRNAs. Science. 309:1514-1518.

2. Keene, J.D. 2007. RNA regulons: coordination of post-transcriptional events. Nat. Rev. Genet. 8:533-543.

3. Bhattacharyya, S.N., and Filipowicz, W. 2007. Argonautes and company: sailing against the wind. Cell. 128:1027-1028.

4. Bhattacharyya, S.N., Habermacher, R., Martine, U., Closs, E.I., and Filipowicz, W. 2006. Relief of microRNA-mediated translational repression in human cells subjected to stress. Cell. 125:1111-1124.

5. Abdelmohsen, K., Lal, A., Kim, H.H., and Gorospe, M. 2007. Posttranscriptional orchestration of an anti-apoptotic program by HuR. Cell Cycle. 6:1288-1292.

6. Brennan, C.M., and Steitz, J.A. 2001. HuR and mRNA stability. Cell. Mol. Life Sci. 58:266-277.

7. Gorospe, M. 2003. HuR in the mammalian genotoxic response: post-transcriptional multitasking. Cell Cycle. 2:412-414.

8. Dixon, D.A., et al. 2001. Altered expression of the mRNA stability factor HuR promotes cyclooxygenase- 2 expression in colon cancer cells. J. Clin. Invest. 108:1657-1665.

9. Sengupta, S., et al. 2003. The RNA-binding protein HuR regulates the expression of cyclooxygenase- 2 . J. Biol. Chem. 278:25227-25233.

10. Mazan-Mamczarz, K., et al. 2003. RNA-binding protein HuR enhances p53 translation in response to ultraviolet light irradiation. Proc. Natl. Acad. Sci. U. S. A. 100:8354-8359.

11. Zou, T., et al. 2006. Polyamine depletion increases cytoplasmic levels of RNA-binding protein HuR leading to stabilization of nucleophosmin and p53 mRNAs. J. Biol. Chem. 281:19387-19394.

12. Tong, X., and Pelling, J.C. 2009. Enhancement of p53 expression in keratinocytes by the bioflavonoid apigenin is associated with RNA-binding protein HuR. Mol. Carcinog. 48:118-129.

13. Erkinheimo, T.L., et al. 2003. Cytoplasmic HuR expression correlates with poor outcome and with cyclooxygenase 2 expression in serous ovarian carcinoma. Cancer Res. 63:7591-7594.

14. Heinonen, M., et al. 2005. Cytoplasmic HuR expression is a prognostic factor in invasive ductal breast carcinoma. Cancer Res. 65:2157-2161.

15. Mrena, J., et al. 2005. Cyclooxygenase-2 is an independent prognostic factor in gastric cancer and its expression is regulated by the messenger RNA sta-

Received for publication December 8, 2008, and accepted in revised form August 26, 2009.

Address correspondence to: Timothy Hla, Center for Vascular Biology, Department of Pathology and Laboratory Medicine, Weill Cornell Medical College, Box 69, 1300 York Avenue, New York, New York, USA. Phone: (212) 746-9953; Fax: (212) 746-2830; E-mail: tih2002@ med.cornell.edu. Or to: Hector Leonardo Aguila, Department of Immunology, University of Connecticut Health Center, 263 Farmington Ave., Farmington, Connecticut 06030, USA. Phone: (860) 679-7658; Fax: (860) 679-8130; E-mail: aguila@nso1.uchc.edu.

bility factor HuR. Clin. Cancer Res. 11:7362-7368.

16. Katsanou, V., et al. 2005. HuR as a negative posttranscriptional modulator in inflammation. Mol. Cell. 19:777-789.

17. Levadoux-Martin, M., et al. 2003. Impaired gametogenesis in mice that overexpress the RNA-binding protein HuR. EMBO Rep. 4:394-399.

18. Katsanou, V., et al. 2009. The RNA-binding protein Elavl1/HuR is essential for placental branching morphogenesis and embryonic development. Mol. Cell. Biol. 29:2762-2776.

19. Tang, S.H., Silva, F.J., Tsark, W.M., and Mann, J.R. 2002. A Cre/loxP-deleter transgenic line in mouse strain 129S1/SvImJ. Genesis. 32:199-202.

20. Takeda, K., Cowan, A., and Fong, G.H. 2007. Essential role for prolyl hydroxylase domain protein 2 in oxygen homeostasis of the adult vascular system. Circulation. 116:774-781.

21. Morrison, S.J., and Weissman, I.L. 1994. The longterm repopulating subset of hematopoietic stem cells is deterministic and isolatable by phenotype. Immunity. 1:661-673.

22. Spangrude, G.J., Heimfeld, S., and Weissman, I.L. 1988. Purification and characterization of mouse hematopoietic stem cells. Science. 241:58-62.

23. Kondo, M., et al. 2000. Cell-fate conversion of lymphoid-committed progenitors by instructive actions of cytokines. Nature. 407:383-386.

24. Hardy, R.R., and Hayakawa, K. 2001. B cell development pathways. Annu. Rev. Immunol. 19:595-621.

25. Akashi, K., Traver, D., Miyamoto, T., and Weissman, I.L. 2000. A clonogenic common myeloid progenitor that gives rise to all myeloid lineages. Nature. 404:193-197.

26. Jacquin, C., Gran, D.E., Lee, S.K., Lorenzo, J.A., and Aguila, H.L. 2006. Identification of multiple osteoclast precursor populations in murine bone marrow. J. Bone Miner. Res. 21:67-77.

27. Kina, T., et al. 2000. The monoclonal antibody TER-119 recognizes a molecule associated with glycophorin A and specifically marks the late stages of murine erythroid lineage. Br. J. Haematol. 109:280-287.

28. Zuniga-Pflucker, J.C., and Lenardo, M.J. 1996. Regulation of thymocyte development from immature progenitors. Curr. Opin. Immunol. 8:215-224.

29. Vermes, I., Haanen, C., Steffens-Nakken, H., and Reutelingsperger, C. 1995. A novel assay for apoptosis. Flow cytometric detection of phosphatidylserine expression on early apoptotic cells using fluorescein labelled Annexin V. J. Immunol. Methods. 184:39-51.

30. Christensen, J.L., and Weissman, I.L. 2001. Flk-2 is a marker in hematopoietic stem cell differentiation: a simple method to isolate long-term stem cells. Proc. Natl. Acad. Sci. U. S. A. 98:14541-14546.

31. Calvano, S.E., et al. 2005. A network-based analysis of systemic inflammation in humans. Nature. 437:1032-1037.

32. Levine, A.J., Hu, W., and Feng, Z. 2006. The P53 pathway: what questions remain to be explored? Cell Death Differ. 13:1027-1036.

33. Montgomery, R.K., and Breault, D.T. 2008. Small intestinal stem cell markers. J. Anat. 213:52-58.

34. Sharpless, N.E. 2005. INK4a/ARF: a multifunctional tumor suppressor locus. Mutat. Res. 576:22-38.

35. Blackshear, P.J. 2002. Tristetraprolin and other $\mathrm{CCCH}$ tandem zinc-finger proteins in the regulation of mRNA turnover. Biochem. Soc. Trans. 30:945-952.

36. Dean, J.L., Sully, G., Clark, A.R., and Saklatvala, J. 2004. The involvement of AU-rich element-binding proteins in p38 mitogen-activated protein kinase pathway-mediated mRNA stabilisation. Cell Signal. 16:1113-1121.

37. Saklatvala, J., Dean, J., and Clark, A. 2003. Control of the expression of inflammatory response genes. Biochem. Soc. Symp. 70:95-106.

38. Lopez de Silanes, I., Lal, A., and Gorospe, M. 2005. HuR: post-transcriptional paths to malignancy. RNA Biol. 2:11-13.

39. Kim, H.H., et al. 2009. HuR recruits let-7/RISC to repress c-Myc expression. Genes Dev. 23:1743-1748.

40. Ghosh, M., et al. 2007. COX-2 suppresses tissue factor expression via endocannabinoid-directed PPARdelta activation. J. Exp. Med. 204:2053-2061.

41. Kondo, M., Weissman, I.L., and Akashi, K. 1997. Identification of clonogenic common lymphoid progenitors in mouse bone marrow. Cell. 91:661-672.

42. Takeda, K., et al. 2008. Regulation of adult erythropoiesis by prolyl hydroxylase domain proteins. Blood. 111:3229-3235.

43. Sun, H., et al. 1999. PTEN modulates cell cycle progression and cell survival by regulating phosphatidylinositol 3,4,5,-trisphosphate and Akt/protein kinase B signaling pathway. Proc. Natl. Acad. Sci.U.S.A. 96:6199-6204.

44. Abdelmohsen, K., et al. 2007. Phosphorylation of HuR by Chk2 regulates SIRT1 expression. Mol. Cell. 25:543-557. 